\title{
UN CONJUNTO MONETARIO DEL SIGLO IV ENCONTRADO EN LA CALLE ARMIÑÁN DE RONDA (MÁLAGA)*
}

\author{
A SET OF COINS FROM THE FOURTH CENTURY FOUND IN THE CALLE ARMIÑÁN OF RONDA \\ (MÁLAGA)
}

JOSÉ ORTIZ CÓRDOBA

Universidad de Granada

joseortiz@ugr.es

https://orcid.org/0000-0003-3737-1115

Recepción: 31-03-2018

Aceptación: 04-06-2018

\section{Resumen}

Presentamos un conjunto de 245 monedas acuñadas en el siglo IV d.C. procedente de las excavaciones urbanas realizadas en la Calle Armiñán de Ronda entre finales de los años 90 y comienzos de la década de los 2000. Las piezas que lo componen permiten encuadrarlo entre los conjuntos teodosianos de estructura corta. Fue abandonado a comienzos del siglo V d.C. tras derrumbarse la vivienda en la que se encontraba depositado como consecuencia de un incendio fortuito.

Palabras clave. Ronda; Calle Armiñán; Circulación monetaria; Antigüedad Tardía; $A E 2$.

\begin{abstract}
We present a set of 245 coins minted in the 4th century $\mathrm{AD}$ from the urban excavations carried out in the Armiñán street in Ronda between the late 90s and the early 2000s. The pieces that make it up allow it to be framed among the short-range Theodosian sets. It was abandoned at the beginning of the 5 th century $\mathrm{AD}$ after the house in which it was deposited collapsed as a consequence of an accidental fire.
\end{abstract}

Key words. Ronda; Calle Armiñán; Monetary circulation; Late Antiquity; AE2.

\footnotetext{
* Este trabajo se enmarca dentro de las líneas de estudio del grupo de investigación HUM-215 dirigido por el Prof. Dr. C. González Román y forma parte de la tesis doctoral Las colonias romanas de Hispania y los movimientos de población (Siglos I-II d.C.) que actualmente desarrollamos en la Universidad de Granada gracias a un contrato FPU otorgado por el Ministerio de Educación, Cultura y Deporte.
} 


\section{INTRODUCCIÓN}

Los trabajos arqueológicos llevados a cabo desde los años 80 del siglo pasado en el casco histórico de Ronda han proporcionado, entre otros hallazgos, un notable volumen de material numismático procedente, en su inmensa mayoría, de época tardía. Se trata de un número algo superior a 300 monedas que han permanecido inéditas en su mayor parte hasta el momento. Con el fin de cubrir esta laguna iniciamos hace algunos años, en común acuerdo con el Museo de Ronda, el estudio y catalogación de esas monedas, actualmente expuestas en la nueva sala dedicada a la Antigüedad Tardía abierta en el museo (Fig. 1). Fruto de estos trabajos vio la luz en el año 2015 una primera contribución que daba a conocer un total de 64 monedas altoimperiales $\mathrm{y}$ tardoantiguas procedentes de varias intervenciones arqueológicas realizadas en Ronda entre los años 90 y comienzos de los 2000 (Ortiz Córdoba, 2015). Ese trabajo se completa hoy con el estudio de las monedas restantes, un total de 245 piezas, pertenecientes todas ellas a un mismo conjunto monetario encontrado en la céntrica Calle Armiñán. Quisiera aprovechar estas líneas para mostrar mi sincero agradecimiento al Museo de Ronda, especialmente a José Manuel Castaño Aguilar, por la cesión de la documentación, las grandes facilidades dadas para el estudio de la misma y su ayuda constante durante todo este tiempo.

\section{MARCO ARQUEOLÓGICO}

La ciudad de Ronda ocupa el lugar sobre el que antaño se alzara la romana Arunda, mencionada por Plinio (NH, III, 14) y Ptolomeo (Geographia, II, 4, 11) en sus respectivas obras y cuyo topónimo conocemos también a través de la epigrafía (CIL II, 1359 y 1360). Estaríamos en un primer momento ante uno más de los 120 oppida stipendiaria que Plinio enumeró para la Bética, aunque con posterioridad la ciudad alcanzaría el rango municipal en época Flavia (Morales Rodríguez, 2000: 68). Por todo ello no puede dudarse del emplazamiento en este solar de la romana Arunda. Sin embargo, los restos romanos presentes en la ciudad siempre han sido escasos y solo comenzaron a emerger de forma parcial a partir de los años 80 del siglo pasado. Esta particularidad se debe a la continua ocupación de la ciudad, que ha provocado notables cambios en su fisonomía urbana. Particularmente importante fue la apertura en el último tercio del siglo XVIII de la Calle Armiñán, un gran vial que atraviesa de norte a sur el actual casco antiguo. Su realización supuso la reordenación del trazado de las calles que afectaban a ese nuevo eje, así como un corte radical en la estructura de la ladera. $\mathrm{La}$ operación afectó notablemente a las casas orientadas hacia la nueva vía, obligando a los inmuebles situados en su lado Oeste a vaciar todo el relleno arqueológico previo para poder alcanzar la cota de altura de la nueva calle (Castaño Aguilar et al., 2005: 48). Como es de suponer, este proceso de vaciado arqueológico afectó de forma notable a lo largo de toda la calle a los restos de época medieval y romana, que fueron sistemáticamente desmontados. Este vaciado venía a sumarse a los ya practicados en épocas anteriores, que también afectaron a los restos romanos y protohistóricos localizados en esta parte de la ciudad. Así pues, los testimonios arqueológicos de época romana sacados a la luz en las distintas excavaciones urbanas, exceptuando la basílica paleocristiana encontrada en la parte más alta de la ciudad (Adroher Auroux et al., 1993), son de escasa

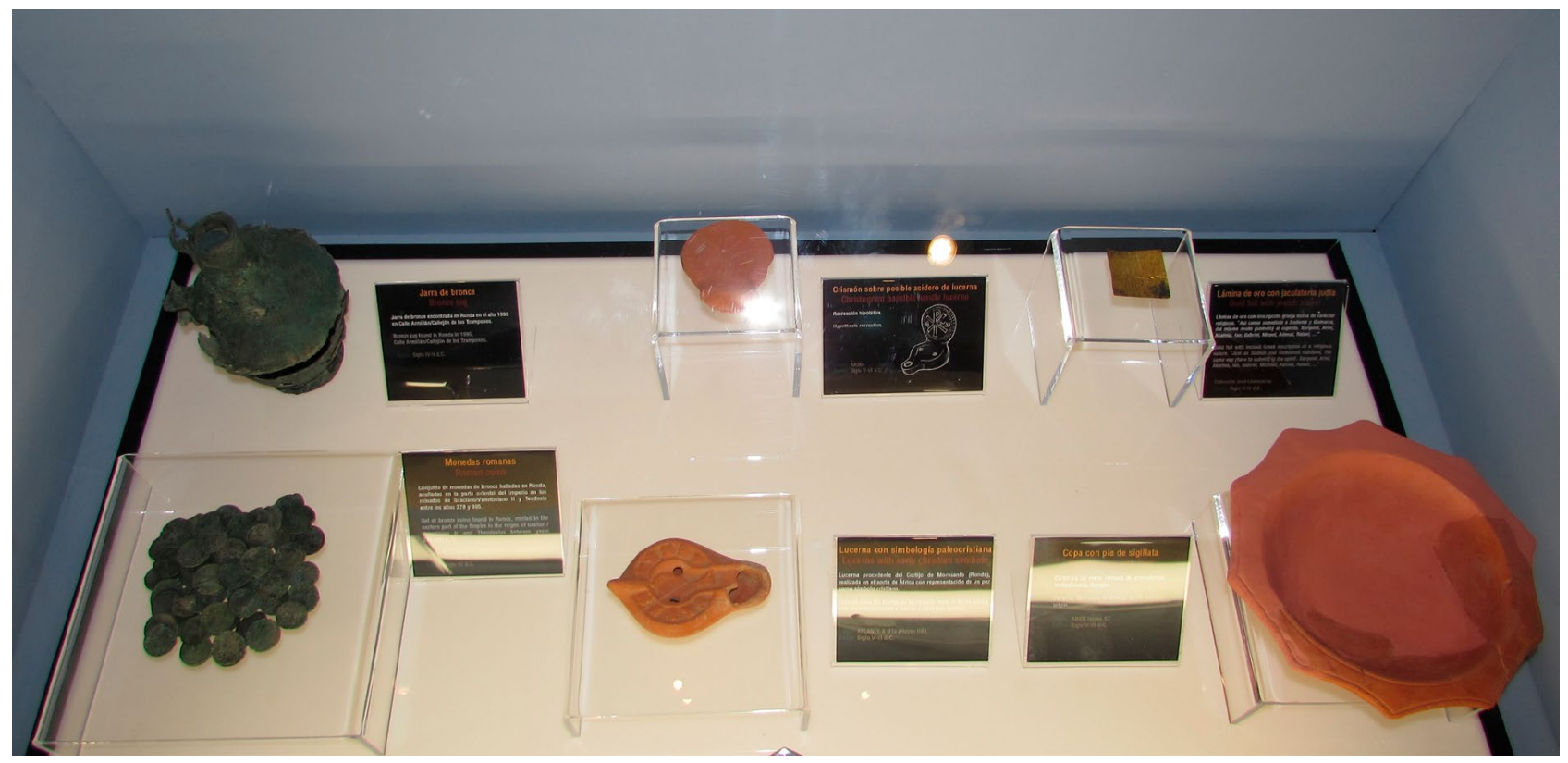

Figura 1: Materiales de época tardía expuestos en la sala dedicada a la Antigüedad Tardía del Museo de Ronda (Imagen: José Manuel Castaño Aguilar). 


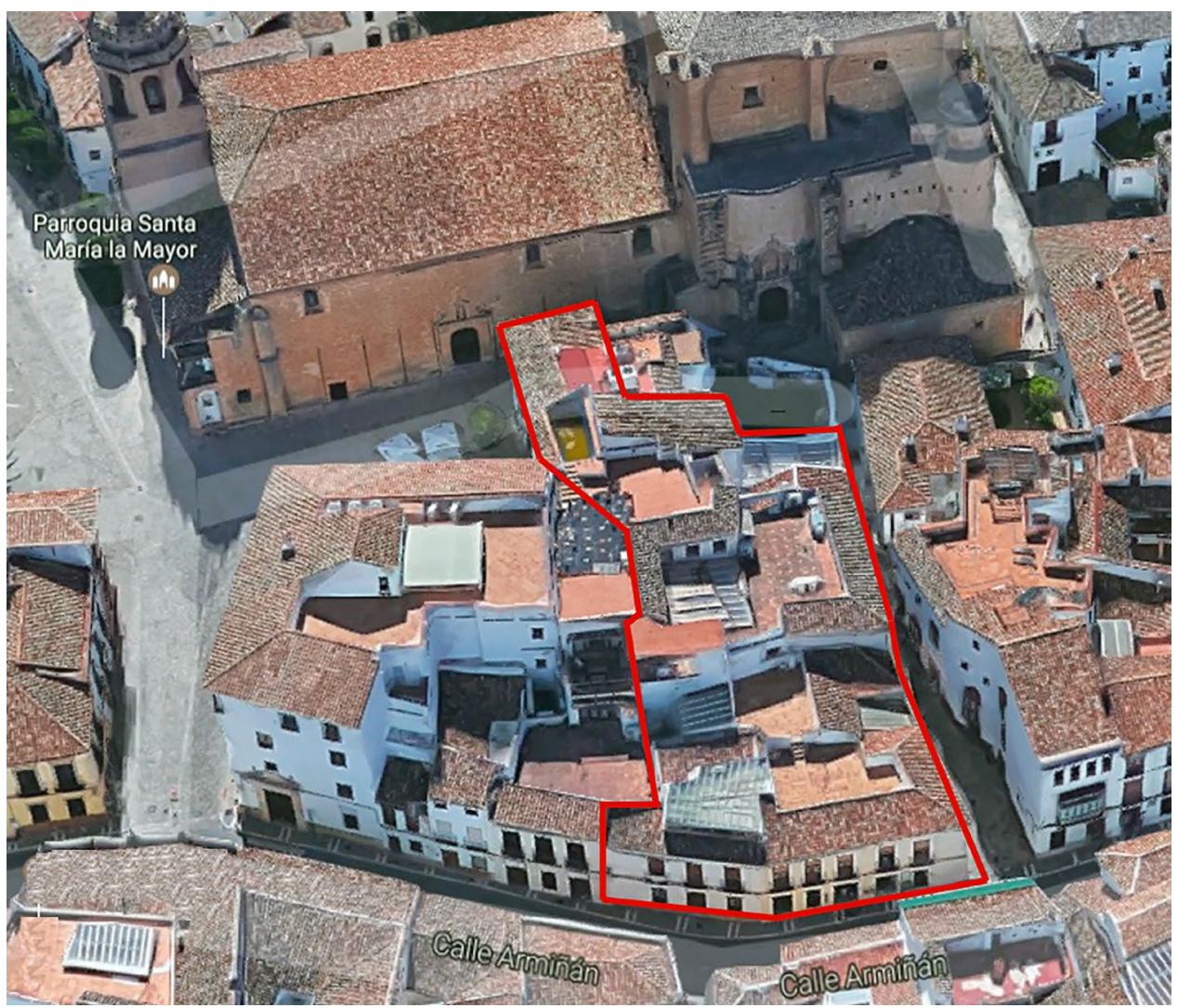

Figura 2: Solares excavados en el periodo 1994-2000 en la Calle Armiñán de Ronda (Elaboración propia).

entidad y se vinculan principalmente a recintos domésticos, careciendo hasta el momento de indicios fiables que nos permitan localizar los espacios públicos de la ciudad romana.

Las monedas que aquí presentamos proceden precisamente de las intervenciones realizadas en distintas zonas de la Calle Armiñán, varios de cuyos solares han aportado hasta ahora los restos romanos mejor conocidos de la ciudad. Los trabajos fueron llevados a cabo en la manzana urbana comprendida entre la Plaza Duquesa de Parcent, el Pasaje Vicente Becerra, el Callejón de los Tramposos y la Calle Armiñán (Fig. 2). En sus diferentes solares se realizaron entre los años 1994 y 2000 una serie de excavaciones de urgencia que aportaron interesante información sobre la evolución urbana de la ciudad (Aguayo de Hoyos et al., 2004). Concretamente, fueron sacadas a la luz diversas estructuras domésticas de época altoimperial dispuestas en torno a una calle, constatando así un uso principalmente residencial para esta zona de la meseta. Junto al material cerámico de esta fase, caracterizado por producciones comunes, sigillatae hispánicas e importaciones de cerámicas finas de mesa, apareció un lote de unas veinticinco monedas mal conservadas que correspondían en su mayoría al siglo III d.C. y que dimos a conocer en el estudio anteriormente mencionado. No obstante, fueron identificadas también varias piezas del siglo II d.C. La época de circulación de estas monedas fue tomada como término final para esta fase urbana (Ortiz Córdoba, 2015: catálogo de monedas, n. ${ }^{\circ} 20$ y 46).
A partir de siglo III d.C. la zona sufre una importante transformación que trae consigo el cambio de funcionalidad de algunos espacios. Se procede a la privatización de la calle, que a partir de ahora pasará a formar parte de un patio empedrado en torno al que se dispusieron una serie de estancias domésticas, unas de carácter fructuario y otras de tipo residencial. Esta peculiar disposición fue identificada con la estructura de una villa urbana donde se reunían producción y residencia y entre cuyos restos fue encontrado el lote de 245 monedas bajoimperiales que presentamos en este trabajo. Junto a este importante número de monedas aparecieron sigillatae africanas claras, objetos suntuarios de bronce y diversos fragmentos de mármol y enlucido. Según pudo constatarse, el edificio sufrió un colapso brusco como consecuencia de un incendio que provocó el derrumbe de sus techos y sepultó este conjunto monetario en una de las estancias de la residencia. Dicho suceso habría tenido lugar en la primera mitad del siglo $\mathrm{V}$ en función de la fecha aportada por las monedas encontradas, los fragmentos cerámicos presentes y los datos derivados de una prueba de Carbono 14 obtenida de una de las vigas quemadas (Aguayo de Hoyos, et al., 2004: 783). Tras el incendio no se produjo ningún intento de recuperar los restos sepultados, quedando este sector de la ciudad como una zona de escombros sobre los que se superpusieron las nuevas construcciones de época medieval. Los hallazgos monetarios de esta fase arqueológica se completan con la aparición de forma dispersa en otras zonas de la manzana de diversas monedas acuñadas en el siglo IV d.C. 


\section{LA DOCUMENTACIÓN NUMISMÁTICA}

El conjunto monetario que estudiamos está compuesto por un total de 245 piezas pertenecientes todas ellas al siglo IV d.C. (Fig. 3). Su aparición fue dada a conocer por sus excavadores en la publicación de los resultados arqueológicos de las campañas realizadas en la Calle Armiñán entre los años 1994 y 2000, aunque carecía hasta el momento de un estudio en profundidad (Aguayo de Hoyos, et al., 2004: 781-782. También reseñado en Nieto González, 2007: 120-121). Debe indicarse igualmente que el conjunto fue excavado durante dos campañas distintas comprendidas entre los años 1995 y 1997. Este hecho queda reflejado en las distintas tablas de datos que figuran al final de este trabajo (Fig. 12), donde existen dos numeraciones de registro diferentes (Ro-95-art y Ro-97-art) que hacen referencia a esos dos años. Se trata en cualquier caso de una cuestión metodológica relacionada con los distintos momentos en que fueron excavados los solares de esta manzana, ya que nos encontramos ante un único conjunto monetario.

Cronológicamente la mayor parte de este conjunto presenta una horquilla temporal bastante bien definida. La misma se sitúa durante el siglo IV d.C., concretamente en la franja temporal que transcurre entre los reinados de los emperadores Graciano/Valentiniano II y Teodosio, esto es, entre los años 378 y 395 d.C. según la cronología que se desprende de las monedas estudiadas. No obstante, conviene señalar también la aparición de cuatro piezas que fueron acuñadas con anterioridad a ese periodo. Se trata de monedas puestas en circulación por los emperadores Diocleciano, Maximino Daia, Constante y Decentio.

En lo que se refiere al estado de conservación de las monedas estudiadas podemos decir que, en general, todas ellas presentan un estado de conservación aceptable que en algunos casos llega incluso a ser bastante bueno. No obstante, otras muchas piezas muestran claras señales de desgaste en, al menos, una de sus caras y otras tantas presentan roturas y concreciones que ha sido imposible eliminar. Estas circunstancias, unidas al desgaste general que muestran todas las monedas, han dificultado las labores de análisis y clasificación, exigiendo un minucioso trabajo de limpieza en muchas de ellas.

Por lo que respecta a los valores presentes, constatamos un abrumador predominio de $A E 2$, emitidos mayoritariamente por Teodosio y sus hijos, Arcadio y Honorio. A ellos hay que sumar las piezas de este mismo valor emitidas por los emperadores Graciano, Valentiniano II y Magno Máximo. Así, sobre un total de 245 monedas, encontramos $160 A E 2$, lo que supone un $65,30 \%$ del total del conjunto. Junto a ellas hemos documentado también un AE Follis emitido por Maximino Daia (312-313), una Fracción de Radiado de Diocleciano emitida en 303 d.C., un $A E 3$ de Decencio (350-353 d.C) y, finalmente, un $A E 4$ del emperador Constante (¿337-347? d.C.). Las restantes 81 piezas no han podido ser identificadas con seguridad; no obstante, por su tamaño, su peso y por los tipos monetales que presentan sus reversos, puede inferirse que casi todas ellas pertenecían también al valor $A E 2$ mayoritario en el conjunto.

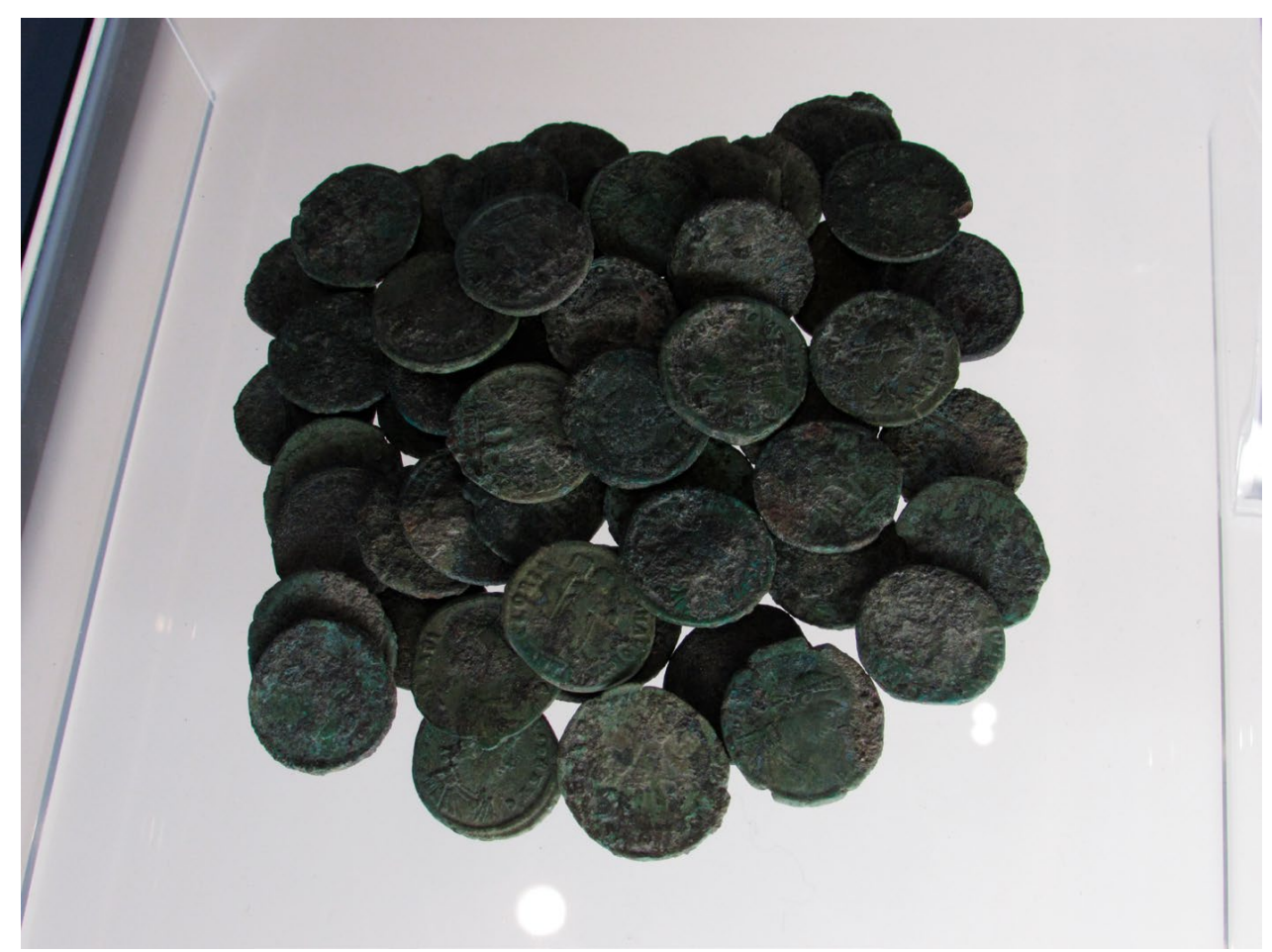

Figura 3: Detalle del conjunto monetario tal y como ha quedado expuesto en la sala dedicada a la Antigüedad Tardía del Museo de Ronda (Imagen: José Manuel Castaño Aguilar). 


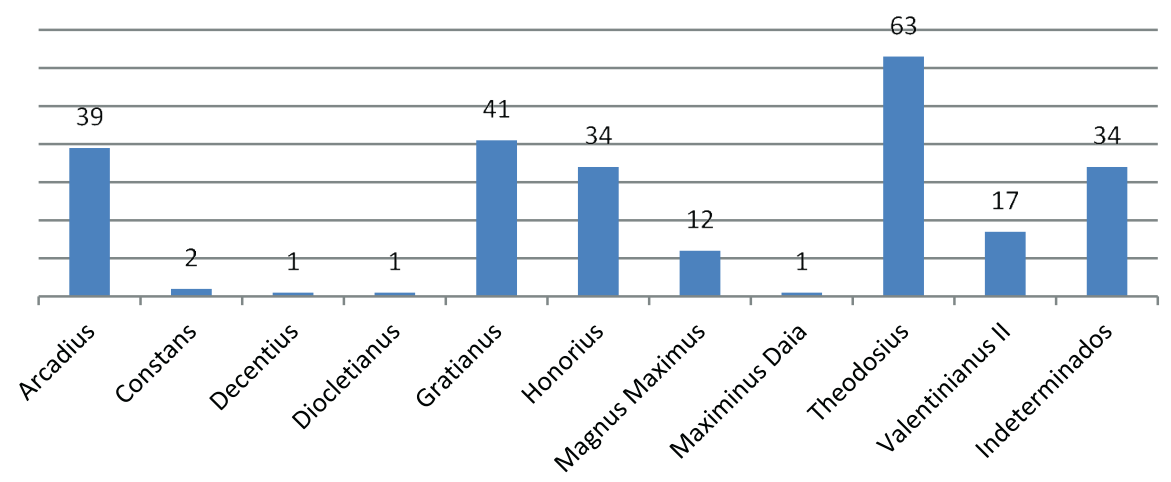

Figura 4: Emperadores representados.

Los emperadores representados se elevan a un total de diez (Fig. 4). De ellos Teodosio es el que más monedas aporta con un total de 63 (el 25,71\% de la muestra). Le siguen Graciano con 41 piezas $(16,73 \%)$ y los emperadores Arcadio y Honorio con 39 y 34 monedas respectivamente $(15,91 \%$ y $13,87 \%$ de la muestra). Aparecen también representados, aunque con un volumen notablemente menor, otros soberanos: Valentiniano II (17 piezas), Magno Máximo (12), Constante (2) y Diocleciano, Maximino Daia y Decentio (una emisión cada uno). Hay además un notable número de monedas (34, un $13,87 \%$ del total) donde no ha podido ser identificado el emperador emisor debido al desgaste o mal estado que presentan las mismas.

En lo que se refiere a los reversos constatamos la presencia de hasta ocho tipos diferentes, si bien los que aparecen con más frecuencia en el conjunto son GLORIA ROMANORVM -emperador estante a derecha portando globo y lábaro (Fig. 5) - y REPARATIO $R E I P V B$-emperador estante a izquierda portando Victoria sobre globo. Ofrece su mano a una figura femenina tocada con corona mural que está arrodillada a su izquierda (Fig. 6)-, tal y como puede observarse en la figura 7. En ella constatamos la mayoritaria presencia en el conjunto de los GLORIA ROMANORVM con un total de 127 monedas $(51,83 \%)^{1}$. Estas acuñaciones proceden en exclusiva de las cecas orientales de Antioquía, Nicomedia, Constantinopla, Cízico, Heraclea, Siscia y Alejandría, donde fueron puestas en circulación por los emperadores Teodosio, Arcadio y Honorio en el periodo 392-395 d.C. En segundo lugar se sitúan las piezas con reverso REPARATIO REIPVB, cuyo número asciende a 91 monedas $(37,14 \%)$. Estas proceden fundamentalmente de cecas situadas en la pars occidentalis del Imperio como Arelate, Roma, Lugdunum y Tréveris. No obstante, este tipo también aparece acuñado, aunque en un volumen mucho menor,

1. Todas ellas pertenecen al tipo emperador estante a derecha portando globo y lábaro, salvo una. Esta pieza, la número 173 de la tabla adjunta (Fig. 12), es la única que se sale de este patrón. En su reverso podemos ver la siguiente composición: Emperador estante a la derecha, con pie sobre cautivo. Porta globo y lábaro. en algunas cecas de la pars orientalis. Así, lo tenemos constatado en Antioquía, Aquileia, Nicomedia, Siscia, Constantinopla y Tesalónica. Fue puesto en circulación mayoritariamente por Graciano y Valentiniano II en Occidente para el periodo 378-383 d.C., para el que también se han constatado varios ejemplares acuñados por Teodosio en cecas orientales. Por su parte, Magno Máximo las acuñó en el periodo 383-388 d.C. en las cecas occidentales de Arelate, Lugdunum y Roma.

El resto de tipos representados lo hacen en escaso número. Destaca la presencia de tres monedas con leyenda VIRTVS EXERCITI -emperador estante a derecha, con pie sobre cautivo. Porta globo y lábaroprocedentes de las cecas de Antioquía, donde fue acuñado por Arcadio en 383-388, y Nicomedia, donde fueron puestos en circulación por Valentiniano II y Teodosio en el mismo periodo cronológico. Por lo demás, debe reseñarse la aparición de los tipos VICTORIAE DD NN AVG ET CAES -dos Victorias sosteniendo escudo con inscripción en 4 líneas $V O T$ $V$-MVLT-X-, acuñado por Decentio en Lugdunum
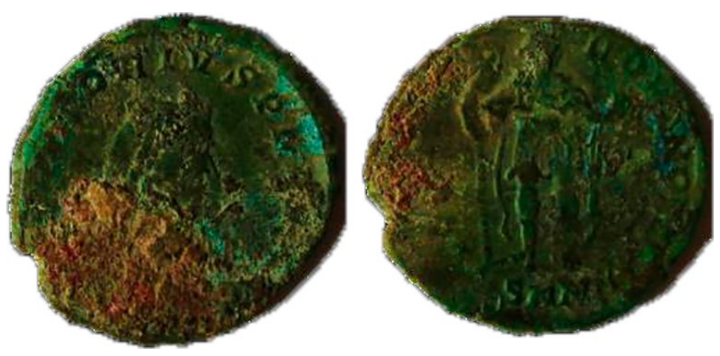

Figura 5: $A E 2$ acuñado por Honorio en Cízico con leyenda GLORIA ROMANORVM (392-395 d.C.) (RIC IX, 46c).
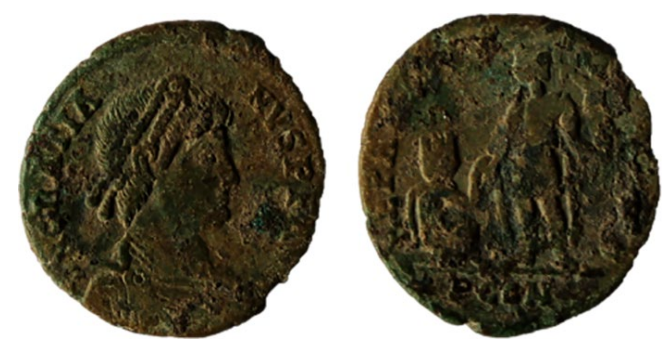

Figura 6: $A E 2$ acuñado por Graciano en Arelate con leyenda REPARATIO REIPVB (378-383 d.C.) (RIC IX, 20a). 


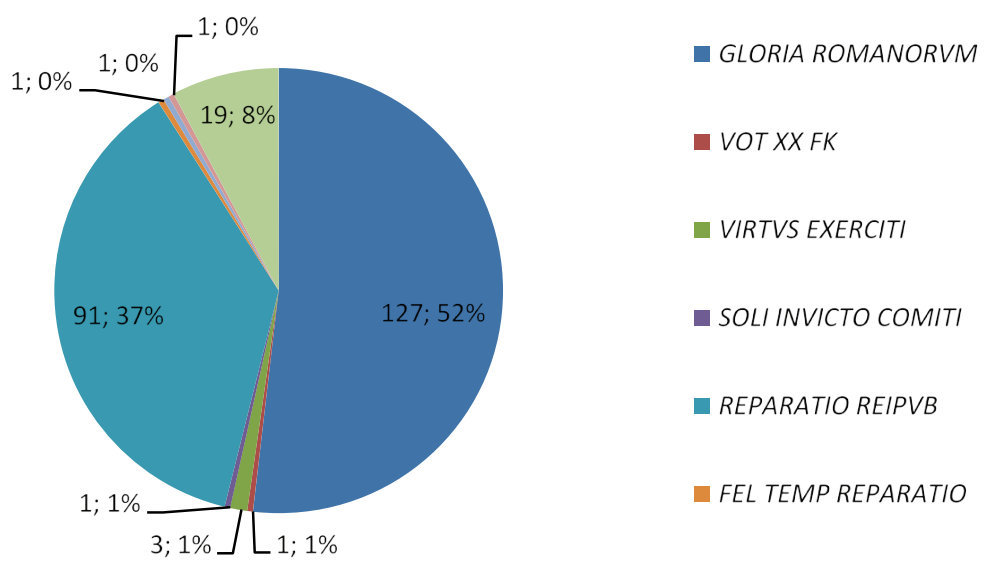

Figura 7: Reversos.

(350-353 d.C.), SOLI INVICTO COMITI, -Sol de pie, a derecha, con clámide sobre el hombro izquierdo, sostiene un globo en su mano izquierda y levanta la mano derecha-, emitido por Maximino Daia en Roma (312313 d.C.) y GLORIA EXERCITVS -dos soldados en pie con lanza y escudo. Entre ellos, un estandarte militar-y FEL TEMP REPARATIO -emperador a izquierda, con uniforme militar, de pie sobre galera sosteniendo un fénix en el globo y lábaro. Victoria sentada en la popa gobernando el buque-, batidos por Constante sin que podamos precisar ni su ceca ni su cronología debido al mal estado de ambas monedas. Junto a ellas debe señalarse también la existencia de 19 piezas cuyos reversos han aparecido frustros o se encuentran tan desgastados que resultan imposibles de identificar.

Por último, en cuanto a las cecas presentes en el conjunto (Fig. 8), constatamos un total de catorce. De ellas, nueve se encuentran en la parte oriental del Imperio y seis lo hacen en su zona occidental. Cuantitativamente la ceca más destacada es Antioquía, que aporta un total de 38 piezas $(15,51 \%)$. De ellas, 30 ejemplares pertenecen al tipo GLORIA ROMANORVM del periodo 392-395 d.C. y otras 7 al tipo REPARATIO REIPVB, con una cronología de 373-383 d.C. La pieza restante está asociada al tipo VIRTVS EXERCITI y fue emitida por Arcadio con fecha 383-388 d.C. Todas las monedas identificadas para esta ceca pertenecen al valor $A E 2$ predominante en este conjunto.

La segunda ceca que más piezas aporta es la de Nicomedia, con 29 monedas $(11,83 \%)$. En ella volvemos a encontrar el tipo GLORIA ROMANORVM como el más representado con 22 piezas. Le siguen a mucha distancia los tipos REPARATIO REIPVB (cinco monedas) y VIRTVS EXERCITI con dos piezas, emitidas por Valentiniano II y Teodosio (Fig. 9).

En tercer lugar se encuentran los talleres de Constantinopla y Arlés, que aportan 22 piezas cada uno $(8,97 \%)$. En el caso de Constantinopla el tipo dominante vuelve a ser el GLORIA ROMANORVM con 20 piezas, mientras que el REPARATIO REIPVB

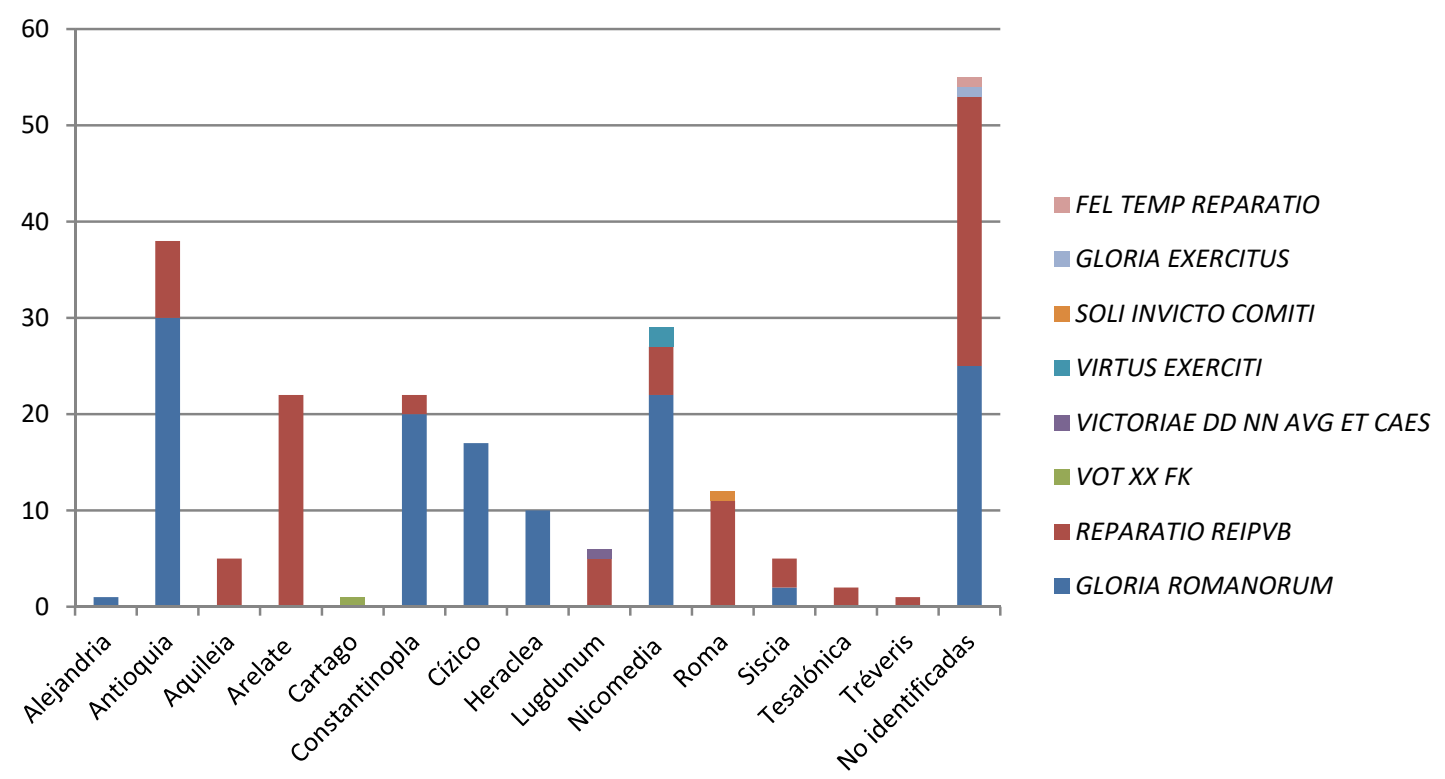

Figura 8: Cecas documentadas y distribución de reversos por cecas. 

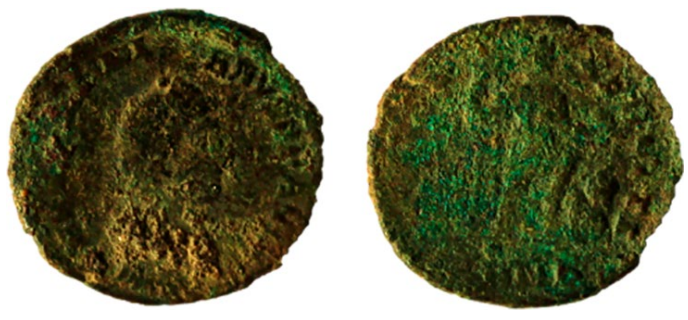

Figura 9: $A E 2$ acuñado por Valentiniano II en Nicomedia con leyenda VIRTVS EXERCITI (383-388 d.C.) (RIC IX, 44a).

únicamente aparece documentado en dos monedas. Por su parte, en Arlés sólo ha sido constatado el tipo REPARATIO REIPVB, que copa de esta manera las 22 piezas procedentes de esta ceca.

Cízico y Heraclea son otros de los talleres que encontramos en este conjunto aportando 17 y 10 monedas respectivamente. En ambos casos únicamente se constatan monedas del tipo GLORIA ROMANORVM. Todo lo contrario ocurre en las cecas de Aquileia (5 piezas), Tesalónica (2) y Tréveris (1), pues en todas ellas sólo han sido constatadas acuñaciones con reverso REPARATIO REIPVB.

Por su parte, las oficinas de Roma, Lugdunum y Siscia presentan una mayoría del tipo REPARATIO $R E I P V B$, aunque en ellas también se documentan otras piezas. En el caso de la capital imperial once de las doce piezas documentadas han sido acuñadas con el tipo REPARATIO REIPVB. La moneda restante, batida por Maximino Daia, porta la efigie del Sol y la leyenda SOLI INVICTO COMITI (Fig. 10). Un patrón similar se observa en Lugdunum, donde cinco monedas corresponden al tipo REPARATIO REIPVB, mientras que la sexta pieza documentada corresponde en este caso a un $A E 3$ del emperador Decentio con la leyenda VICTORIAE DD NN AVG ET CAES (350-353 d.C.). Por su parte, en Siscia hemos documentado la presencia de tres piezas con el tipo REPARATIO REIPVB y otras dos correspondientes al tipo GLORIA ROMANORVM.

Debe reseñarse también la aparición de varios talleres cuya presencia es casi testimonial en este conjunto. Se trata de los casos de Alejandría, con una única moneda perteneciente al tipo GLORIA ROMANORVM, y Cartago, donde igualmente solo se documenta una pieza. En este caso se trata de una fracción de radiado
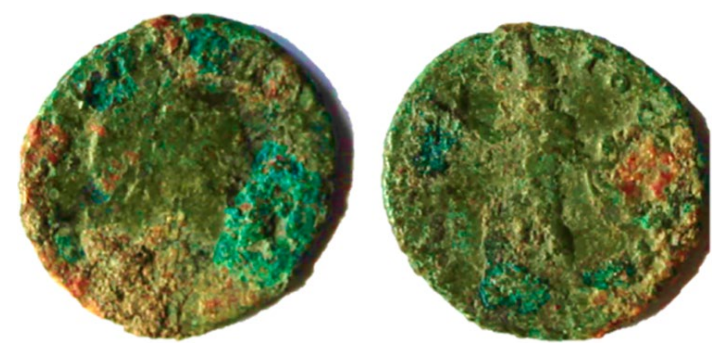

Figura 10: $A E 2$ acuñado por Maximiano Daia en Roma con leyenda SOLI INVICTO COMITI (312-313 d.C.) (RIC VI, 335b).
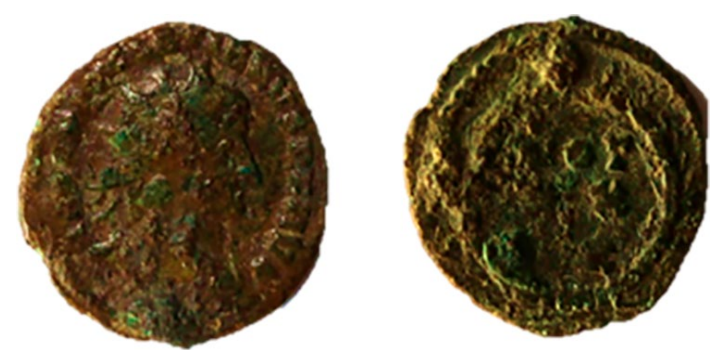

Figura 11: Fracción de radiado acuñada por Diocleciano en Cartago con leyenda VOT XX FK (303 d.C.) (RIC VI, 38).

fechada en el año 303 d.C. y emitida por el emperador Diocleciano con leyenda VOT XX FK (Fig. 11).

Conviene mencionar igualmente la existencia de un elevado número de piezas, un total de 74 , un $30,20 \%$, cuya ceca no hemos podido identificar. De ellas, 28 piezas pertenecen al tipo REPARATIO REIPVB, 25 al tipo GLORIA ROMANORVM, una pieza al tipo GLORIA EXERCITVS y otra ha sido identificada como un FEL TEMP REPARATIO emitido por el emperador Constante.

Por último, y para finalizar, del estudio de las cecas del conjunto se desprende que la mayor parte de las monedas documentadas -129 sobre un total de 245 , un $52,65 \%$ - proceden de talleres situados en la Pars Orientalis del Imperio, constatando así la relación de aprovisionamiento monetario de la Península Ibérica con esta zona del Imperio durante el siglo IV d.C. (Cepeda Ocampo, 2000: 166).

\section{VALORACIÓN}

En primer lugar debemos reseñar que las especiales circunstancias arqueológicas del casco urbano de Ronda - continua ocupación habitacional desde época pre y protohistórica y existencia de varios procesos de vaciado arqueológico- han condicionado, lógicamente, el material numismático de que disponemos en la actualidad. Este se encuentra, asimismo, estrechamente relacionado con los escasos restos romanos sacados a la luz hasta el momento y que pertenecen mayoritariamente a época tardía, como ya hemos indicado. Por ello conviene señalar que aunque son numerosas las referencias que encontramos en autores locales de los siglos XVI-XVIII al hallazgo en el casco antiguo de Ronda de monedas de época republicana y altoimperial (Sobre estos autores: Ortiz Córdoba, 2017: 199-215), la realidad es que las piezas de que disponemos actualmente pertenecen, salvo contadas excepciones, a época tardía. En este sentido, Arunda presenta un notable contraste con la vecina Acinipo, ciudad con la que debió mantener una estrecha relación dada su cercanía. Arunda, además de no disponer de una ceca propia, apenas documenta monedas de época republicana o altoimperial, al contrario de lo que sucede en Acinipo, donde éstas son muy abundantes (Mora Serrano y Ojeda Marín, 1988: 593-600; Mora 
Serrano, 1988: 89-100; Mora Serrano, 1990: 3-12; Mora Serrano, 1999: 341-348; Mora Serrano, 2001: 427-430; Ortiz Córdoba (en prensa). Esta cuestión debe también ponerse en relación, además de con las especiales circunstancias arqueológicas de Ronda, con el propio proceso histórico que vive la región en época imperial. El hecho de que Acinipo fuese durante el Principado la ciudad más importante de la Depresión de Ronda, concentrando por ello la mayor parte de la población de la zona y experimentando un notable desarrollo urbano y económico, debió incidir lógicamente en la cantidad y calidad del numerario presente en la ciudad. Arunda, sin embargo, experimenta el proceso contrario, pasando de ocupar un lugar secundario durante los primeros siglos del Principado a convertirse en el centro hegemónico de la Depresión a partir del siglo IV d.C. y en adelante, momento al que pertenecen la gran mayoría de las monedas documentadas en la ciudad.

Un segundo elemento a tener en cuenta es que la composición y cronología del conjunto que damos a conocer ponen este hallazgo en relación con otros existentes en la provincia de Málaga, donde distintas contribuciones han resaltado el importante volumen de monedas del siglo IV d.C. recuperadas en los distintos yacimientos de la provincia. Esta cuestión estaría estrechamente relacionada con el aumento de la moneda en circulación en Hispania para esta época y, desde una perspectiva arqueológica, con la mayor facilidad de acceso a los niveles bajoimperiales (Mora Serrano, 2001: 442). Entre estos conjuntos malagueños, sin ánimo de ser exhaustivos, podemos citar los procedentes de los yacimientos de la villa romana de Manguarra y San José (Cártama), donde destaca una importante presencia de $A E 2$ de los tipos REPARATIO REIPVB y GLORIA ROMANORVM acuñados entre los años 378-395 (Mora Serrano, 1993: 133-145), y la villa romana de Sabinillas (Manilva), en cuya composición destaca la presencia de moneda de mediados del siglo IV d.C. junto a los mayoritarios AE2 de época de Teodosio (Rodríguez Oliva, 1979: 835-852). Igualmente interesantes son los hallazgos monetarios procedentes del teatro romano de Málaga; en este caso su fecha de acuñación no suele ir más allá del siglo IV d.C., aunque una parte importante de la moneda documentada debió seguir circulando durante los siglos V y VI d.C. (Mora Serrano, 2003: 359-376; Mora Serrano, 2007: 191-212). Finalmente, dentro de los hallazgos documentados en Andalucía podemos destacar el llevado a cabo en el Cortijo Chirino, en las cercanías de Écija, que presenta importantes similitudes en cuanto a cronología y composición con el conjunto rondeño (Chaves Tristán, et al., 2000: 465-475).

A su vez, las monedas encontradas en Ronda, tanto las estudiadas en un anterior trabajo ya mencionado, como las presentadas aquí, reproducen el patrón de una importante mayoría de yacimientos hispanos. En ellos, el periodo que aporta un mayor número de ejemplares es el que transcurre entre la segunda mitad del siglo III y comienzos del siglo V d.C. en un marco de predominio de la moneda de bronce (San Vicente, 1999: 707-708). En este sentido, el patrón del conjunto monetario aquí estudiado reproduce casi fielmente lo ya expuesto para otros conjuntos peninsulares (para algunos ejemplos: San Vicente, 1999: 675-691; Cepeda Ocampo, 2000: 162-165), a saber, la presencia y predominio de dos grandes bloques monetarios correspondientes, uno de ellos, a los AE2 REPARATIO REIPVB emitidos por Graciano, Valentiniano II y Teodosio y completados por la serie del mismo reverso que acuña Magno Máximo en varias cecas de la Galia, y el otro a la serie GLORIA ROMANORVM -emperador con globo y lábaro- batida en las cecas orientales por los miembros de la Casa de Teodosio entre el 392 y el 395. Ambos bloques responderían a dos contextos distintos de producción que la fase final de tesaurización ha mezclado (Cepeda Ocampo, 2000: 165; Chaves Tristán, et al., 2000: 468-469).

Por último, debe hacerse una referencia al carácter del hallazgo. En este sentido creemos que, a tenor de la documentación arqueológica, no debemos considerar este conjunto monetario como un tesorillo $\mathrm{u}$ ocultamiento voluntario. Más bien al contrario, las circunstancias del hallazgo nos inclinan a pensar en un hecho fortuito. Sabemos que en la estancia donde aparecieron las monedas se encontró también un objeto metálico de bronce destinado probablemente a contenerlas (Aguayo de Hoyos, et al., 2004: 782) (Fig. 2). De hecho, la localización de las piezas, esparcidas a lo largo de toda la estancia, hace pensar que las mismas estarían situadas en un lugar visible, probablemente una estantería, de la que cayeron como consecuencia del hundimiento de la techumbre. El colapso brusco que parece sufrir el edificio a raíz del incendio anteriormente referido sepultó la totalidad del contenido de la estancia. Este hecho tuvo lugar a comienzos del siglo $\mathrm{V}$, pudiendo fijarse su fecha en el 410 d.C. a partir de las pruebas de Carbono 14 realizada a una de las vigas del conjunto (Aguayo de Hoyos, et al., 2004: 783). Esta fecha tiene, sin duda, unas connotaciones icónicas en la historia de Roma, pero la realidad es que nada nos indica en el resto del panorama arqueológico de Ronda o la Serranía la presencia de episodios violentos para este periodo. Por tanto, pensamos que debió tratarse de un hecho fortuito, quizás un accidente, producto de un incendio producido en algunas de las estancias del complejo, fenómeno nada extraño en las ciudades antiguas. Este hecho, unido a la ausencia de circunstancias de inestabilidad, nos induce a pensar que no estaríamos, por tanto, ante un ocultamiento voluntario de estas monedas. Sin embargo, sí llama la atención y por el momento no tenemos respuesta para ello, el que los dueños del solar no retornaran al mismo una vez sofocado el incendio, quedando esta zona como una escombrera que no sería ocupada de nuevo hasta época altomedieval. 


\section{CATÁLOGO DE LAS MONEDAS}

\begin{tabular}{|c|c|c|}
\hline \multicolumn{3}{|c|}{ CONJUNTO MONETARIO DEL SIGLO IV d.C. PROCEDENTE DE RONDA. CATÁLOGO DE MONEDAS } \\
\hline Abreviaturas & Tipos de Anversos & Tipos de Reverso \\
\hline $\begin{array}{l}\text { N-1: Número Orden } \\
\text { N.R.A: Número de registro Arqueológico } \\
\text { A.T.: Tipo de Anverso } \\
\text { R.T.: Tipo de Reverso } \\
\text { C: Posición de cuño (en horas) } \\
\text { M: Módulo (en milímetros) } \\
\text { P: Peso (en gramos) } \\
\text { CRON.: Cronología } \\
\text { R.I.C.: Ref. a The Roman Imperial } \\
\text { Coinage }\end{array}$ & $\begin{array}{l}\text { B1: Busto diademado, manto, coraza, a } \\
\text { derecha } \\
\text { B2: Busto a derecha, desnudo, coraza } \\
\text { B3: Busto diademado a derecha, lanza, } \\
\text { escudo. Manus Dei portando una } \\
\text { guirnalda, en la parte superior del campo. } \\
\text { B4: Busto diademado, manto, coraza, a } \\
\text { izquierda } \\
\text { B5: Busto radiado, manto, coraza, a } \\
\text { derecha } \\
\text { B6: Busto laureado, coraza, a derecha }\end{array}$ & $\begin{array}{l}\text { R1: Emperador de pie a derecha con } \\
\text { globo y lábaro } \\
\text { R2: Emperador coronado por Victoria } \\
\text { ayudando a levantarse a Mujer con corona } \\
\text { mural } \\
\text { R3: Dos Victorias sosteniendo escudo con } \\
\text { inscripción en } 4 \text { líneas VOT-V-MVLT-X } \\
\text { R4: Emperador de frente con lábaro y } \\
\text { escudo. Cautivo arrodillado a sus pies } \\
\text { R5: Dos soldados en pie con lanza y } \\
\text { escudo. Entre ellos, un estandarte militar } \\
\text { R6: Emperador a izquierda, con uniforme } \\
\text { militar, de pie sobre galera sosteniendo } \\
\text { un fénix en el globo y lábaro. Victoria } \\
\text { sentada en la popa gobernando el buque } \\
\text { R7: Emperador estante a derecha, con pie } \\
\text { sobre cautivo. Porta globo y lábaro } \\
\text { R8: Inscripción votiva VOT XX FK dentro } \\
\text { de corona, en tres líneas } \\
\text { R9: Sol de pie, a derecha, con clámide } \\
\text { sobre el hombro izquierdo, sostiene un } \\
\text { globo en su mano izquierda y levanta la } \\
\text { mano derecha }\end{array}$ \\
\hline
\end{tabular}

\begin{tabular}{|c|c|c|c|c|c|c|c|c|c|c|c|c|c|}
\hline & \multicolumn{10}{|c|}{ ALEJANDRIA } & CRON. & VALOR & R.I.C. \\
\hline N-1 & N.R.A & A.T. & LEYENDA DE ANVERSO & R.T. & LEYENDA DE REVERSO & EXERGO & CAMPO & C & M & P & CRO \\
\hline 1 & $\begin{array}{c}\text { Ro-97-art } \\
143.379(66)\end{array}$ & B1 & (dn) HONORIVS PF AVG & R1 & GLORIA-(ro)MANORVM & ALEA & & 11 & 21.5 & 4.21 & $392-395$ & AE2 & IX, 21d \\
\hline
\end{tabular}

\begin{tabular}{|c|c|c|c|c|c|c|c|c|c|c|c|c|c|}
\hline & \multicolumn{13}{|c|}{ ANTIOQUIA } \\
\hline $\mathrm{N}-1$ & N.R.A & A.T. & LEYENDA DE ANVERSO & R.T. & LEYENDA DE REVERSO & EXERGO & CAMPO & $\mathrm{C}$ & M & $P$ & CRON. & VALOR & R.I.C. \\
\hline 2 & $\begin{array}{c}\text { Ro-95-art } \\
141.386\end{array}$ & B1 & (dn theodo)-SIVS (pf avg) & R1 & GLO(ria)-ROM(ano)RVM & ANTA & & 6 & 22 & 5.12 & $392-395$ & AE2 & $\mathrm{IX}, 68 \mathrm{a}$ \\
\hline 3 & \begin{tabular}{|l|} 
Ro-95-art \\
$141.390(4)$ \\
\end{tabular} & B1 & DN ARCADI-VS PF AVG & R1 & (gloria)-ROMANORVM & ANTB & & 11 & 21.8 & 5.04 & $392-395$ & AE2 & IX, $68 \mathrm{c}$ \\
\hline 4 & \begin{tabular}{|c|} 
Ro-95-art \\
$141.390(9)$
\end{tabular} & $\mathrm{B} 1$ & (dn ho)NORIVS PF AV(g) & $\mathrm{R} 1$ & GLORIA-(roman)ORVM & ANTГ & & 4 & 23.6 & 4.57 & $392-395$ & AE2 & IX, $68 \mathrm{e}$ \\
\hline 5 & \begin{tabular}{|c|} 
Ro-95-art \\
$141.390(16)$ \\
\end{tabular} & B1 & $\begin{array}{c}\text { DN VALENTINIANVS } \\
\mathrm{P}(\mathrm{f} \text { avg })\end{array}$ & $\mathrm{R} 2$ & (repa)RATIO-REIPVB & ANTB & & 11 & 22.7 & 5.73 & $378-383$ & AE2 & IX, $42 \mathrm{c}$ \\
\hline 6 & \begin{tabular}{|c|} 
Ro-95-art \\
$141.415(21)$ \\
\end{tabular} & B1 & (dn the)ODO-SIVS PF AVG & R1 & (gloria)-ROMANORVM & ANT $\Delta$ & & 11 & 21.8 & 4.33 & $392-395$ & AE2 & IX, 68a \\
\hline 7 & \begin{tabular}{|c|} 
Ro-95-art \\
$141.415(23)$ \\
\end{tabular} & B1 & (dn theodo)-SIVS PF AVG & R1 & G(loria)-(romano)RVM & ANTA & & 4 & 22.5 & 3.81 & $392-395$ & AE2 & $\mathrm{IX}, 68 \mathrm{a}$ \\
\hline 8 & \begin{tabular}{|c|} 
Ro-95-art \\
$141.415(26)$ \\
\end{tabular} & B1 & DN THEODO-(sivs pf avg) & R2 & REPARAT(io)-REIPVB & ANTГ & & 12 & 24.5 & 5.25 & 378-383 & $\mathrm{AE} 2$ & IX, $42 \mathrm{~d}$ \\
\hline 9 & \begin{tabular}{|c|} 
Ro-95-art \\
$141.415(29)$
\end{tabular} & $\mathrm{B} 1$ & (dn theodo)-SIV & $\mathrm{R} 1$ & (gl)ORIA-ROMANORVM & ANTA & & 4 & 20 & 3.98 & $392-395$ & AE2 & IX, 68a \\
\hline 10 & $\begin{array}{l}\text { Ro-95-art } \\
141.452(1)\end{array}$ & B1 & DN AR(cadi)-VS PF AVG & R1 & $\begin{array}{l}\text { (g)LORIA-(r) } \\
\text { OMANORVM }\end{array}$ & ANTГ & & 6 & 20.5 & 4.65 & $392-395$ & AE2 & IX, 68d \\
\hline 11 & \begin{tabular}{|l|} 
Ro-95-art \\
$141.452(2)$ \\
\end{tabular} & B1 & DN THEODO-SIV(s pf avg) & R1 & (gloria)-ROMAN(orv)M & ANT $\Delta$ & & 4 & 19.5 & 4.39 & $392-395$ & AE2 & IX, 68a \\
\hline 12 & \begin{tabular}{|l|} 
Ro-95-art \\
$141.452(3)$ \\
\end{tabular} & B1 & (dn theodo)-SIVS PF AVG & R1 & GLORIA-(romanorvm) & ANTA & & 5 & 20 & 4.69 & $392-395$ & $\mathrm{AE} 2$ & IX, 68a \\
\hline 13 & \begin{tabular}{|c|} 
Ro-95-art \\
$141.526(1)$ \\
\end{tabular} & B1 & DN TH(eodo)-(sivs pf avg) & R1 & GLORIA-(romanorv)M & ANT $\Delta$ & & 11 & 21 & 4.48 & $392-395$ & $\mathrm{AE} 2$ & IX, 68a \\
\hline 14 & \begin{tabular}{|c|} 
Ro-95-art \\
$141.526(3)$ \\
\end{tabular} & B1 & (dn a)RCADI-VS PF AVG & R1 & GLORIA-ROMANO(rvm) & $\mathrm{AN}(\mathrm{t}-)$ & & 11 & 22 & 4.73 & $392-395$ & AE2 & IX, $68 \mathrm{c}$ \\
\hline 15 & \begin{tabular}{|c|} 
Ro-95-art \\
$141.526(5)$ \\
\end{tabular} & B1 & DN GRATIA-NVS PF AVG & $\mathrm{R} 2$ & REPARATIO-RE(ipvb) & ANTA & & 12 & 24 & 4.88 & $378-383$ & AE2 & $\mathrm{IX}, 42 \mathrm{a}$ \\
\hline 16 & \begin{tabular}{|c|} 
Ro-95-art \\
$141.526(12)$ \\
\end{tabular} & B1 & DN ARCADI-VS PF AVG & R1 & GLORIA-ROMANORVM & ANTB & & 6 & 22 & 5.01 & $392-395$ & AE2 & IX, $68 \mathrm{c}$ \\
\hline 17 & \begin{tabular}{|c|} 
Ro-95-art \\
$141.526(19)$
\end{tabular} & B1 & $\begin{array}{l}\text { DN THEODO-SIVS PF } \\
\text { AVG }\end{array}$ & R1 & GLORIA-ROMANORVM & ANTA & & 5 & 22.5 & 5.89 & $392-395$ & AE2 & $\mathrm{IX}, 68 \mathrm{a}$ \\
\hline
\end{tabular}




\begin{tabular}{|c|c|c|c|c|c|c|c|c|c|c|c|c|}
\hline 18 & \begin{tabular}{|c|} 
Ro-95-art \\
$141.526(29)$ \\
\end{tabular} & B1 & DN ARCADI-VS PF AV(g) & R1 & (gloria)-ROMA(no)RVM & ANTA & 6 & 21 & 4.63 & $392-395$ & AE2 & IX, 68c \\
\hline 19 & \begin{tabular}{|c|} 
Ro-95-art \\
$141.452(5)$ \\
\end{tabular} & B1 & DN THEODO-SIVS (pf avg) & R1 & GLOR(ia)-(r)OMA(norv)M & ANTГ & 11 & 23.8 & 4.25 & $392-395$ & AE2 & IX, 68a \\
\hline 20 & \begin{tabular}{|c|} 
Ro-95-art \\
$141.458(2)$ \\
\end{tabular} & B1 & (dn hono)RIVS PF A(vg) & R1 & GLORIA-(romanorvm) & ANTA & 11 & 21.2 & 4.38 & $392-395$ & $\mathrm{AE} 2$ & IX, 68e \\
\hline 21 & $\begin{array}{l}\text { Ro-95-art } \\
142.216 \\
\end{array}$ & B1 & DN HONORIVS PF (avg) & R1 & (gloria)-(rom)ANORVM & ANTГ & 5 & 23 & 5.60 & $392-395$ & AE2 & IX, 68e \\
\hline 22 & $\begin{array}{c}\text { Ro-95-art } \\
142.841 \\
\end{array}$ & B1 & DN HONORIVS PF AV(g) & R1 & G(lor)IA-ROMANORVM & ANT $\Delta$ & 11 & 21.8 & 5.28 & $392-395$ & $\mathrm{AE} 2$ & IX, $68 \mathrm{f}$ \\
\hline 23 & $\begin{array}{c}\text { Ro-95-art } \\
142.854 \\
\end{array}$ & B1 & (dn arca)DIVS (pf) AVG & R7 & VIRTVS E-(xerciti) & ANT(s) & 12 & 22 & 3.57 & $383-388$ & AE2 & IX, 63e \\
\hline 24 & \begin{tabular}{|c|} 
Ro-95-art \\
$143.054(1)$ \\
\end{tabular} & B1 & $\begin{array}{c}\text { DN THEODO-SIVS PF } \\
\text { AVG }\end{array}$ & R1 & (glo)RIA-ROM(anorvm) & ANTA & 5 & 22.2 & 4.09 & $392-395$ & AE2 & IX, 68a \\
\hline 25 & \begin{tabular}{|c|} 
Ro-95-art \\
$143.054(2)$ \\
\end{tabular} & B1 & $\begin{array}{l}\text { DN THE(odo)-(si)VS (pf) } \\
\text { AVG } \\
\end{array}$ & R1 & GLOR(ia)-(roma)N(or)VM & (a)NTA & 11 & 21.1 & 4.08 & $392-395$ & AE2 & IX, $68 \mathrm{a}$ \\
\hline 26 & \begin{tabular}{|c|} 
Ro-97-art \\
$143.379(4)$ \\
\end{tabular} & B1 & $\begin{array}{l}\text { DN THEODO-SIVS (PF) } \\
\text { AVG } \\
\end{array}$ & $\mathrm{R} 2$ & REPARATIO-(reip)VB & ANTГ & 5 & 21.8 & 6.10 & $378-383$ & $\mathrm{AE} 2$ & IX, 42d \\
\hline 27 & \begin{tabular}{|c|} 
Ro-97-art \\
$143.379(6)$ \\
\end{tabular} & B1 & $\begin{array}{l}\text { DN THEO(do)-SIVS PF } \\
\text { AV(G) }\end{array}$ & R2 & REPARA(tio)-(reipvb) & ANT(-) & 11 & 23 & 4.41 & $378-383$ & $\mathrm{AE} 2$ & IX, 42d \\
\hline 28 & \begin{tabular}{|c|} 
Ro-97-art \\
$143.379(9)$ \\
\end{tabular} & B1 & (dn) THEODO-(sivs pf avg) & R1 & (gl)OR(i)A-(romanor)VM & ANT(-) & 6 & 21.8 & 4.19 & $392-395$ & AE2 & IX, 68a \\
\hline 29 & \begin{tabular}{|c|} 
Ro-97-art \\
$143.379(10)$ \\
\end{tabular} & B1 & (dn the)ODO-SIVS(pf avg) & R1 & GLORIA-RO(manorvm) & ANTA & 5 & 20 & 5.13 & $392-395$ & AE2 & IX, $68 \mathrm{a}$ \\
\hline 30 & \begin{tabular}{|c|} 
Ro-97-art \\
$143.379(13)$ \\
\end{tabular} & B1 & $\begin{array}{c}\text { (dn) THEODO-(sivs pf a) } \\
\text { V(g) }\end{array}$ & R1 & (gloria-romanorvm) & ANTA & 11 & 21 & 4.27 & $392-395$ & AE2 & IX, $68 \mathrm{a}$ \\
\hline 31 & \begin{tabular}{|c|} 
Ro-97-art \\
143.379(14) \\
\end{tabular} & B1 & $\begin{array}{c}(\mathrm{dn} t) \mathrm{HEOD}(\mathrm{o}) \text {-SIVS PF } \\
\text { (avg) }\end{array}$ & R1 & GLO(ri)A-ROMANORVM & ANTA & 5 & 21.5 & 5.06 & $392-395$ & $\mathrm{AE} 2$ & IX, 68a \\
\hline 32 & \begin{tabular}{|c|} 
Ro-97-art \\
$143.379(15)$ \\
\end{tabular} & B1 & (dn the)ODO-SI(vs pf) AVG & R1 & GLORIA-ROMANORVM & ANT $\Delta$ & 4 & 20.5 & 5.13 & $392-395$ & AE2 & IX, 68a \\
\hline 33 & \begin{tabular}{|c|} 
Ro-97-art \\
$143.379(16)$ \\
\end{tabular} & B1 & (dn theodo)-SIVS PF AVG & R1 & GLORIA-(romanorvm) & ANT(-) & 11 & 21 & 3.95 & $392-395$ & AE2 & IX, 68a \\
\hline 34 & \begin{tabular}{|c|} 
Ro-97-art \\
$143.379(17)$ \\
\end{tabular} & B1 & $\begin{array}{l}\text { DN THEOD(o)-SIVS PF } \\
\text { A(vg) } \\
\end{array}$ & R1 & GLORIA-(r)OMANO(rvm) & ANTA & 6 & 22 & 4.17 & $392-395$ & AE2 & IX, $68 \mathrm{a}$ \\
\hline 35 & \begin{tabular}{|c|} 
Ro-97-art \\
$143.379(24)$ \\
\end{tabular} & B1 & $\begin{array}{c}\text { (dn v)ALENTINI(anvs pf } \\
\text { avg) }\end{array}$ & $\mathrm{R} 2$ & (reparati)O-(reipvb) & ANTB & 11 & 23.8 & 5.76 & $378-383$ & AE2 & IX, 42c \\
\hline 36 & \begin{tabular}{|c|} 
Ro-97-art \\
$143.379(54)$ \\
\end{tabular} & B1 & DN (g)RATIA-NVS PF AVG & R2 & REPA(ratio)-(r)EIPV(b) & ANTA & 4 & 23.5 & 4.85 & $378-383$ & AE2 & $\mathrm{IX}, 42 \mathrm{a}$ \\
\hline 37 & \begin{tabular}{|c|} 
Ro-97-art \\
$143.379(56)$ \\
\end{tabular} & B1 & (dn ho)NORIV(s pf avg) & R1 & GLOR(ia)-(romanorvm) & ANTA & 4 & 23.9 & 3.88 & $392-395$ & AE2 & $\mathrm{IX}, 68 \mathrm{e}$ \\
\hline 38 & \begin{tabular}{|c|} 
Ro-97-art \\
$143.379(57)$ \\
\end{tabular} & B1 & DN HONORIV(s pf avg) & $\mathrm{R} 1$ & GLORIA-(romanorvm) & ANT $\Delta$ & 5 & 23.5 & 4.39 & $392-395$ & AE2 & $\mathrm{IX}, 68 \mathrm{f}$ \\
\hline 39 & \begin{tabular}{|c|} 
Ro-97-art \\
$143.379(65)$ \\
\end{tabular} & B1 & DN HON(orivs pf avg) & R1 & GLORIA-(r)OMANORVM & ANTA & 11 & 22 & 5.50 & 392-395 & $\mathrm{AE} 2$ & IX, 68e \\
\hline
\end{tabular}

\begin{tabular}{|c|c|c|c|c|c|c|c|c|c|c|c|c|c|}
\hline & \multicolumn{13}{|c|}{ AQUILEIA } \\
\hline $\mathrm{N}-1$ & N.R.A & A.T. & LEYENDA DE ANVERSO & R.T. & LEYENDA DE REVERSO & EXERGO & CAMPO & $\mathrm{C}$ & $\mathrm{M}$ & $\mathrm{P}$ & CRON. & VALOR & R.I.C. \\
\hline 40 & \begin{tabular}{|c|} 
Ro-95-art \\
$141.390(17)$ \\
\end{tabular} & B1 & $\begin{array}{l}\text { DN VALENTI(nianvs ivn) } \\
\text { PF AVG }\end{array}$ & R2 & REPAR(atio)-(reipvb) & SMAQP & & 12 & 25 & 4.33 & $378-383$ & AE2 & $\mathrm{IX}, 30 \mathrm{~b}$ \\
\hline 41 & \begin{tabular}{c|} 
Ro-95-art \\
142.843 \\
\end{tabular} & B1 & $\begin{array}{c}\text { DN VALENTINIA(nvs i)VN } \\
\text { P(f av)G }\end{array}$ & R2 & RE(paratio)-REIPVB & SMAQS & & 6 & 25.2 & 5.30 & $378-383$ & AE2 & IX, 30b \\
\hline 42 & \begin{tabular}{|c|} 
Ro-97-art \\
$143.379(12)$ \\
\end{tabular} & B1 & (dn th)EODO-SIVS (pf av)G & $\mathrm{R} 2$ & (r)EPARAT(io)-(reipvb) & SMAQP & & 6 & 22.5 & 4.65 & $378-383$ & AE2 & IX, 30d \\
\hline 43 & \begin{tabular}{|c|} 
Ro-97-art \\
$143.379(20)$ \\
\end{tabular} & B1 & $\begin{array}{c}\text { (dn val)ENTINIANVS(ivn } \\
\text { pf) AVG }\end{array}$ & R2 & (reparatio)-(reipv)B & SMAQS & & 12 & 22.8 & 5.65 & $378-383$ & $\mathrm{AE} 2$ & $\mathrm{IX}, 30 \mathrm{~b}$ \\
\hline 44 & \begin{tabular}{|c|} 
Ro-97-art \\
$143.379(21)$ \\
\end{tabular} & B1 & $\begin{array}{c}\text { DN VALENTINIANVS (i) } \\
\text { VN PF AVG } \\
\end{array}$ & R2 & (reparati)O-(rei)PVB & (s)MAQ & & 6 & 27.9 & 4.82 & $378-383$ & $\mathrm{AE} 2$ & $\mathrm{IX}, 30 \mathrm{~b}$ \\
\hline
\end{tabular}

\begin{tabular}{|c|c|c|c|c|c|c|c|c|c|c|c|c|c|}
\hline & \multicolumn{10}{|c|}{ ARELATE } \\
\hline N-1 & N.R.A & A.T. & LEYENDA DE ANVERSO & R.T. & LEYENDA DE REVERSO & EXERGO & CAMPO & C & M & P & CRON. & VALOR & R.I.C. \\
\hline 45 & $\begin{array}{c}\text { Ro-95-art } \\
141.387(2)\end{array}$ & B1 & DN GRATIA-NVS PF AV(g) & R2 & REPA(ratio)-(reipvb) & PCON & & 5 & 23 & 5.48 & $378-383$ & AE2 & IX, 20a \\
\hline 46 & $\begin{array}{c}\text { Ro-95-art } \\
141.390(5)\end{array}$ & B1 & DN GRATIA-NVS P(f avg) & R2 & (reparat)IO-REIPVB & (-)CON & & 11 & 22.7 & 4.43 & $378-383$ & AE2 & IX, 20a \\
\hline 47 & $\begin{array}{c}\text { Ro-95-art } \\
141.390(14)\end{array}$ & B1 & $\begin{array}{c}\text { DN MAG MAXI-(mvs) PF } \\
\text { AVG }\end{array}$ & R2 & (r)EPAR(atio)-REIPVB & $(-)$ CON & & 11 & 24.5 & 4.54 & $383-388$ & AE2 & IX, 26a \\
\hline 48 & $\begin{array}{c}\text { Ro-95-art } \\
141.390(15)\end{array}$ & B1 & $\begin{array}{c}\text { DN MAG MAXI-(mvs pf } \\
\text { avg) }\end{array}$ & R2 & (re)PARAT(io)-REIPVB & $(-)$ CON & & 6 & 23 & 3.66 & $383-388$ & AE2 & IX, 26a \\
\hline 49 & $\begin{array}{c}\text { Ro-95-art } \\
141.415(3)\end{array}$ & B1 & DN GRATIA-NVS PF A(vg) & R2 & (re)PAR(atio)-(reipvb) & TCON & & 5 & 23.7 & 4.69 & $378-383$ & AE2 & IX, 20a \\
\hline 50 & $\begin{array}{c}\text { Ro-95-art } \\
141.415(5)\end{array}$ & B1 & DN GRATIA-(nvs pf avg) & R2 & R(eparat)IO-REI(pvb) & PCON & & 5 & 26 & 4.88 & $378-383$ & AE2 & IX, 20a \\
\hline
\end{tabular}




\begin{tabular}{|c|c|c|c|c|c|c|c|c|c|c|c|c|}
\hline 51 & \begin{tabular}{|c|} 
Ro-95-art \\
$141.415(13)$ \\
\end{tabular} & B1 & $\begin{array}{l}\text { (dn) MAG MAXI-(mvs } \\
\text { pf avg) }\end{array}$ & R2 & REPA(ratio)-(reip)VB & PCON & 11 & 24 & 4.49 & $383-388$ & AE2 & IX, 26a \\
\hline 52 & \begin{tabular}{|c|} 
Ro-95-art \\
$141.415(18)$ \\
\end{tabular} & B1 & $\begin{array}{c}\text { DN MA(g maxi)-MVS P(f } \\
\text { avg) }\end{array}$ & R2 & REPARATIO-(rei)PVB & SCON & 7 & 22.5 & 3.23 & $383-388$ & AE2 & IX, 26a \\
\hline 53 & \begin{tabular}{|c|} 
Ro-95-art \\
$141.415(24)$ \\
\end{tabular} & B1 & DN GRATIA-(nvs pf avg) & R2 & REPA(ratio)-REIPVB & PCON & 12 & 23.5 & 5.53 & $378-383$ & AE2 & IX, 20a \\
\hline 54 & \begin{tabular}{|c|} 
Ro-95-art \\
$141.415(35)$ \\
\end{tabular} & B1 & (...)S PF (avg) & R2 & (rep)ARATIO-REIPVB & PCON & 11 & 24 & 5.84 & & & \\
\hline 55 & \begin{tabular}{|c|} 
Ro-95-art \\
$141.415(38)$ \\
\end{tabular} & B1 & $\begin{array}{c}\text { DN MAG M(axi)-(mvs pf } \\
\text { avg) }\end{array}$ & R2 & REPARA(tio)-(reipvb) & PCON & 11 & 24 & 4.86 & $383-388$ & AE2 & IX, 26a \\
\hline 56 & \begin{tabular}{|c|} 
Ro-95-art \\
$141.452(7)$ \\
\end{tabular} & B1 & $\begin{array}{c}\text { DN VALENTINIANV(s } \\
\text { pf avg) }\end{array}$ & R2 & REP(arati)O-(reipv)B & PCON & 6 & 22.5 & 4.10 & $378-383$ & $\mathrm{AE} 2$ & IX, 20b \\
\hline 57 & \begin{tabular}{|c|} 
Ro-95-art \\
$141.466(5)$ \\
\end{tabular} & B1 & (dn gratia)-NVS PF AVG & R2 & REPARATIO-(reipvb) & $\mathrm{TC}(\mathrm{on})$ & 10 & 24.2 & 3.68 & $378-383$ & $\mathrm{AE} 2$ & IX, 20a \\
\hline 58 & \begin{tabular}{|c|} 
Ro-95-art \\
$141.526(9)$ \\
\end{tabular} & B1 & & R2 & (repar)ATIO-(reipvb) & PCON & 12 & 23.5 & 5.17 & & & \\
\hline 59 & \begin{tabular}{|c|} 
Ro-95-art \\
$141.526(17)$ \\
\end{tabular} & B1 & (d)N (gr)ATIA-(nvs pf) AVG & R2 & (rep)ARAT(io)-REIPVB & $(-) \mathrm{CON}$ & 5 & 24 & 4.31 & $378-383$ & $\mathrm{AE} 2$ & IX, 20a \\
\hline 60 & \begin{tabular}{|c|} 
Ro-95-art \\
$141.526(21)$ \\
\end{tabular} & B1 & & R2 & REPARATIO-(reip)VB & TCON & 12 & 24 & 4.44 & & & \\
\hline 61 & \begin{tabular}{|c|} 
Ro-95-art \\
$142.215(2)$ \\
\end{tabular} & B1 & (dn gratia)-NVS (pf avg) & R2 & REP(aratio)-(reipvb) & $(-) \mathrm{CON}$ & 6 & 21.5 & 3.70 & $378-383$ & $\mathrm{AE} 2$ & IX, 20a \\
\hline 62 & $\begin{array}{l}\text { Ro-95-art } \\
142.797 \\
\end{array}$ & B1 & $\begin{array}{c}\text { DN VALENTINIANVS IVN } \\
\text { (pf avg) }\end{array}$ & R2 & REPA(ratio)-(reipvb) & PCON & 6 & 23 & 4.36 & $378-383$ & AE2 & IX, 20c \\
\hline 63 & $\begin{array}{l}\text { Ro-95-art } \\
142.844 \\
\end{array}$ & B1 & (dn gr)ATIA-(nvs pf avg) & $\mathrm{R} 2$ & REPARATIO-(reipvb) & PCON & 6 & 24.2 & 3.74 & $378-383$ & AE2 & IX, 20a \\
\hline 64 & \begin{tabular}{c|} 
Ro-95-art \\
143.053 \\
\end{tabular} & B1 & $\begin{array}{c}\text { DN VALEN(tinia)NVS IVN } \\
\mathrm{P}(\mathrm{f} \text { avg })\end{array}$ & R2 & RE(paratio)-(r)EIPVB & $(-) \mathrm{CON}$ & 5 & 23.5 & 4.17 & $378-383$ & $\mathrm{AE} 2$ & IX, 20c \\
\hline 65 & \begin{tabular}{|c|} 
Ro-97-art \\
$143.379(44)$ \\
\end{tabular} & B1 & (dn gr)ATIA-NVS PF (avg) & R2 & (re)PA(ratio)-(r)EIPVB & $\mathrm{TC}(\mathrm{o}) \mathrm{N}$ & 5 & 23.1 & 5.36 & $378-383$ & $\mathrm{AE} 2$ & IX, 20a \\
\hline 66 & \begin{tabular}{|c|} 
Ro-97-art \\
$143.379(55)$
\end{tabular} & B1 & DN GRATIA-(nvs pf avg) & R2 & (reparati)O-(reipvb) & PCON & 5 & 23 & 5.02 & $378-383$ & AE2 & IX, 20a \\
\hline
\end{tabular}

\begin{tabular}{|c|c|c|c|c|c|c|c|c|c|c|c|c|c|}
\hline & \multicolumn{13}{|c|}{ CARTAGO } \\
\hline $\mathrm{N}-1$ & N.R.A & A.T. & LEYENDA DE ANVERSO & R.T. & $\begin{array}{l}\text { LEYENDA DE } \\
\text { REVERSO }\end{array}$ & EXERGO & САMPO & $\mathrm{C}$ & M & $\mathrm{P}$ & CRON. & VALOR & R.I.C. \\
\hline 67 & $\begin{array}{l}\text { Ro-95-art } \\
142.876\end{array}$ & B5 & $\begin{array}{l}\text { IMP C.DIOCLETIANVS } \\
\text { PF AVG }\end{array}$ & $\mathrm{R} 8$ & VOT XX (f)K & & & 12 & 21 & 2.88 & 303 & \begin{tabular}{|c} 
Fracción \\
de \\
radiado
\end{tabular} & VI, 38 \\
\hline
\end{tabular}

\begin{tabular}{|c|c|c|c|c|c|c|c|c|c|c|c|c|c|}
\hline & \multicolumn{13}{|c|}{ CONSTANTINOPLA } \\
\hline $\mathrm{N}-1$ & N.R.A & A.T. & LEYENDA DE ANVERSO & R.T. & LEYENDA DE REVERSO & EXERGO & CAMPO & $\mathrm{C}$ & M & $\mathrm{P}$ & CRON. & VALOR & R.I.C. \\
\hline 68 & \begin{tabular}{|c|} 
Ro-95-art \\
$141.390(25)$ \\
\end{tabular} & B1 & (dn gr)ATIA-(nvs pf avg) & R2 & (reparatio)-REIP(vb) & $\mathrm{C}(\mathrm{ons} \Gamma)$ & & 6 & 21.8 & 4.25 & $378-383$ & AE2 & IX, 54a \\
\hline 69 & \begin{tabular}{|c|} 
Ro-95-art \\
$141.415(2)$ \\
\end{tabular} & B1 & DN THEODO-(sivs pf avg) & $\mathrm{R} 1$ & GLORIA-ROMANORVM & CONS $\Delta$ & & 12 & 21 & 4.41 & $392-395$ & AE2 & IX, 88a \\
\hline 70 & \begin{tabular}{|c|} 
Ro-95-art \\
$141.415(9)$ \\
\end{tabular} & B1 & (dn) HONORIVS PF AV(g) & $\mathrm{R} 1$ & GLOR(ia)-ROMANORVM & CONSA & & 6 & 22.5 & 5.70 & $392-395$ & AE2 & IX, $88 \mathrm{c}$ \\
\hline 71 & \begin{tabular}{|c|} 
Ro-95-art \\
$141.415(11)$ \\
\end{tabular} & B1 & DN (h)ONOR(ivs pf avg) & R1 & GLORIA-ROM(anorvm) & CONSA & & 6 & 22 & 4.69 & $392-395$ & AE2 & IX, $88 \mathrm{c}$ \\
\hline 72 & \begin{tabular}{|c|} 
Ro-95-art \\
$141.415(14)$ \\
\end{tabular} & B1 & $\begin{array}{l}\text { DN THEODO-SIVS (pf } \\
\text { avg) }\end{array}$ & R1 & GLORIA-ROMA(norvm) & CONS $\Delta$ & & 6 & 20.5 & 3.58 & $392-395$ & AE2 & IX, 88a \\
\hline 73 & \begin{tabular}{|c|} 
Ro-95-art \\
$141.415(17)$ \\
\end{tabular} & & & R2 & (reparatio-reipvb) & $\mathrm{CON}(\mathrm{s}-)$ & & & 24.5 & 5.54 & & & \\
\hline 74 & \begin{tabular}{|c|} 
Ro-95-art \\
$141.415(20)$ \\
\end{tabular} & B1 & $\begin{array}{c}\text { (dn t)HEODO-(sivs pf) } \\
\text { AVG }\end{array}$ & R1 & (glo)RIA-ROMANORVM & (co)NSB & & 7 & 20 & 4.81 & $392-395$ & AE2 & IX, 88a \\
\hline 75 & \begin{tabular}{|c|} 
Ro-95-art \\
$141.415(25)$ \\
\end{tabular} & B1 & $\begin{array}{c}\text { DN ARCAD(i)-(vs) PF } \\
\text { AVG }\end{array}$ & R1 & GLORIA-ROMA(no)RVM & $\mathrm{CO}\left(\mathrm{ns}^{-}\right)$ & & 5 & 20.5 & 5.09 & $392-395$ & AE2 & IX, $88 \mathrm{~b}$ \\
\hline 76 & \begin{tabular}{|c|} 
Ro-95-art \\
$141.415(28)$ \\
\end{tabular} & B1 & $\begin{array}{c}\text { DN THEODO-SIVS PF } \\
\text { AVG } \\
\end{array}$ & R1 & GLO(ria)-ROMANORVM & CONSB & & 12 & 21 & 4.65 & $392-395$ & AE2 & IX, 88a \\
\hline 77 & \begin{tabular}{|c|} 
Ro-95-art \\
$141.415(30)$ \\
\end{tabular} & B1 & DN ARCADI-VS PF AVG & R1 & GLORIA-ROMANOR(vm) & CONSГ & & 12 & 21 & 4.28 & $392-395$ & AE2 & IX, 88b \\
\hline 78 & \begin{tabular}{|c|} 
Ro-95-art \\
$141.415(31)$ \\
\end{tabular} & B1 & $\begin{array}{c}\text { DN THEODO-SI(vs pf } \\
\text { avg) }\end{array}$ & $\mathrm{R} 1$ & GLOR(ia)-ROMANORVM & CONSB & & 6 & 20 & 2.92 & $392-395$ & $\mathrm{AE} 2$ & IX, 88a \\
\hline 79 & \begin{tabular}{|c|} 
Ro-95-art \\
$141.415(32)$ \\
\end{tabular} & B1 & (dn a)RCADI-(vs pf avg) & R1 & (gl)ORIA-ROMANORVM & CONSГ & & 6 & 20 & 4.05 & $392-395$ & AE2 & IX, 88b \\
\hline 80 & \begin{tabular}{|l|} 
Ro-95-art \\
$141.526(2)$ \\
\end{tabular} & B1 & DN(theod)O-SIVS PF AVG & $\mathrm{R} 1$ & (glori)A-ROMANORVM & CONSA & & 6 & 21 & 5.03 & $392-395$ & AE2 & IX, 88a \\
\hline 81 & \begin{tabular}{|c|} 
Ro-95-art \\
$141.526(10)$ \\
\end{tabular} & B1 & DN THEODO-(sivs pf avg) & $\mathrm{R} 1$ & GLORIA-(romanorvm) & $\mathrm{CON}(-)$ & & 6 & 22.2 & 4.91 & $392-395$ & $\mathrm{AE} 2$ & IX, 88a \\
\hline
\end{tabular}




\begin{tabular}{|c|c|c|c|c|c|c|c|c|c|c|c|c|c|}
\hline 82 & $\begin{array}{c}\text { Ro-95-art } \\
141.526(18)\end{array}$ & B1 & $\begin{array}{c}\text { DN THE(odo)-SIVS PF } \\
\text { AVG }\end{array}$ & $\mathrm{R} 1$ & GLORIA ROMANORVM & (co)NSB & & 6 & 21 & 3.69 & $392-395$ & AE2 & IX, $88 \mathrm{a}$ \\
\hline 83 & $\begin{array}{l}\text { Ro-95-art } \\
143.055\end{array}$ & $\mathrm{~B} 1$ & & $\mathrm{R} 1$ & (gloria-romanorvm) & (co)NSA & $1+{ }^{2}$ & 6 & 20 & 3.88 & & & \\
\hline 84 & \begin{tabular}{|c|} 
Ro-97-art \\
$143.379(3)$ \\
\end{tabular} & $\mathrm{B} 1$ & $\begin{array}{c}\text { DN (theod)O-SIVS PF } \\
\text { AVG }\end{array}$ & $\mathrm{R} 1$ & GLORIA-RO(man)ORVM & CONS $\Delta$ & & 6 & 21.1 & 3.18 & $392-395$ & AE2 & IX, $88 \mathrm{a}$ \\
\hline 85 & $\begin{array}{c}\text { Ro-97-art } \\
143.379(8)\end{array}$ & $\mathrm{B} 1$ & (dn t)HEODO-(sivs pf avg) & $\mathrm{R} 1$ & (gloria)-(ro)MAN(or)VM & CONSA & & 6 & 22 & 5.95 & $392-395$ & AE2 & IX, $88 \mathrm{a}$ \\
\hline 86 & $\begin{array}{c}\text { Ro-97-art } \\
143.379(19)\end{array}$ & $\mathrm{B} 1$ & $\begin{array}{l}\text { (d)N THEODO-(sivs pf } \\
\text { avg) }\end{array}$ & $\mathrm{R} 1$ & $\begin{array}{c}\text { (g)LORIA-ROM(a)NO(rv) } \\
\mathrm{M}\end{array}$ & CONSB & $1+3$ & 6 & 22 & 4.35 & $392-395$ & AE2 & IX, $88 \mathrm{a}$ \\
\hline 87 & $\begin{array}{c}\text { Ro-97-art } \\
143.379(43)\end{array}$ & B1 & DN ARCADI-(vs pf avg) & $\mathrm{R} 1$ & (gl)ORIA-(romanorv)M & CONST & & 6 & 23.7 & 5.28 & 392-395 & AE2 & $\mathrm{IX}, 88 \mathrm{~b}$ \\
\hline 88 & \begin{tabular}{|c|} 
Ro-97-art \\
$143.379(62)$ \\
\end{tabular} & B1 & $\begin{array}{c}\text { (dn) HONO(ri)VS (pf) } \\
\text { AVG }\end{array}$ & $\mathrm{R} 1$ & GLORIA-ROMANORVM & CONSA & & 6 & 21.5 & 5.73 & 392-395 & $\mathrm{AE} 2$ & IX, $88 \mathrm{c}$ \\
\hline 89 & \begin{tabular}{|c|} 
Ro-97-art \\
$143.379(67)$
\end{tabular} & B1 & $\begin{array}{c}\text { DN (th)EODO-SIVS PF } \\
\text { AVG }\end{array}$ & $\mathrm{R} 1$ & $\begin{array}{l}\text { (g)LOR(ia)-(ro) } \\
\text { MANORVM }\end{array}$ & (co)NSA & $+1+$ & 6 & 21.6 & 3.60 & $392-395$ & AE2 & IX, $88 \mathrm{a}$ \\
\hline
\end{tabular}

\begin{tabular}{|c|c|c|c|c|c|c|c|c|c|c|c|c|c|}
\hline \multirow[b]{2}{*}{$\mathrm{N}-1$} & \multicolumn{13}{|c|}{ CÍZICO } \\
\hline & N.R.A & A.T. & LEYENDA DE ANVERSO & R.T. & LEYENDA DE REVERSO & EXERGO & CAMPO & $\mathrm{C}$ & $\mathrm{M}$ & $P$ & CRON. & VALOR & R.I.C. \\
\hline 90 & \begin{tabular}{|c|} 
Ro-95-art \\
$141.390(6)$ \\
\end{tabular} & B1 & $\begin{array}{l}\text { (d)N THEODO-(sivs pf } \\
\text { avg) }\end{array}$ & $\mathrm{R} 1$ & (g)LOR(ia)-(romanorvm) & SMKA & & 1 & 22 & 5.05 & $392-395$ & AE2 & IX, 27a \\
\hline 91 & $\begin{array}{c}\text { Ro-95-art } \\
141.390(7) \\
\end{array}$ & B1 & $\begin{array}{c}\text { DN HONORIVS (pf) } \\
\mathrm{AV}(\mathrm{g})\end{array}$ & $\mathrm{R} 1$ & (gloria)-(r)OMANORV $(\mathrm{m})$ & $\mathrm{SMK} \Gamma$ & & 12 & 21.5 & 4.38 & $392-395$ & AE2 & IX, 28c \\
\hline 92 & \begin{tabular}{|c|} 
Ro-95-art \\
$141.390(18)$
\end{tabular} & B1 & DN ARCADI-(vs pf avg) & $\mathrm{R} 1$ & G(loria)-(romanorvm) & SMKB & & 11 & 22.5 & 4.48 & $392-395$ & AE2 & IX, 27b \\
\hline 93 & \begin{tabular}{|c|} 
Ro-95-art \\
$141.390(27)$ \\
\end{tabular} & B1 & (dn h)ONORIVS PF (avg) & $\mathrm{R} 1$ & GLO(ria)-ROMANORVM & SMKB & & 6 & 23 & 4.55 & $392-395$ & AE2 & IX, 28c \\
\hline 94 & \begin{tabular}{|c|} 
Ro-95-art \\
$141.415(8)$ \\
\end{tabular} & B1 & DN ARCADI-VS PF AVG & $\mathrm{R} 1$ & (gloria)-ROMANORVM & $\mathrm{S}(\mathrm{m}) \mathrm{KA}$ & & 6 & 23 & 6.21 & $392-395$ & AE2 & IX, 27b \\
\hline 95 & \begin{tabular}{|c|} 
Ro-95-art \\
$141.415(12)$ \\
\end{tabular} & B1 & DN THEODO-(sivs pf avg) & $\mathrm{R} 1$ & GLORIA-(roman)ORVM & (s)MKA & & 6 & 22 & 4.94 & $392-395$ & AE2 & IX, 27a \\
\hline 96 & \begin{tabular}{|c|} 
Ro-95-art \\
$141.415(36)$ \\
\end{tabular} & $\mathrm{B} 1$ & (dn) HONORIVS PF AVG & $\mathrm{R} 1$ & GLORIA-R(omanorvm) & SMKB & & 6 & 22 & 4.39 & $392-395$ & AE2 & IX, 28c \\
\hline 97 & $\begin{array}{c}\text { Ro-95-art } \\
141.515(2)\end{array}$ & B1 & DN ARCADI-VS PF AVG & $\mathrm{R} 1$ & GLO(ria)-ROMANORVM & SMKB & & 12 & 21 & 5.60 & $392-395$ & AE2 & IX, 27b \\
\hline 98 & $\begin{array}{l}\text { Ro-95-art } \\
141.526(6) \\
\end{array}$ & B1 & DN HONORIVS PF (avg) & $\mathrm{R} 1$ & (glor)IA-ROMANORVM & (s)MKB & & 7 & 21 & 3.91 & $392-395$ & AE2 & IX, 28c \\
\hline 99 & \begin{tabular}{|c|} 
Ro-95-art \\
$141.526(13)$ \\
\end{tabular} & B1 & $\begin{array}{c}\text { (dn t)HEODO-SI(vs pf } \\
\text { avg) }\end{array}$ & $\mathrm{R} 1$ & (gloria)-(rom)ANORVM & (s)MKГ & & 12 & 21.5 & 4.62 & $392-395$ & AE2 & IX, 27a \\
\hline 100 & \begin{tabular}{|c|} 
Ro-95-art \\
$141.526(15)$ \\
\end{tabular} & B1 & (dn) HONORIV(s pf avg) & $\mathrm{R} 1$ & GLORIA-ROMANORVM & SMKA & & 7 & 22 & 4.40 & $392-395$ & AE2 & IX, 28c \\
\hline 101 & \begin{tabular}{|c|} 
Ro-95-art \\
$141.526(22)$ \\
\end{tabular} & B1 & $\begin{array}{c}\text { (dn) THE(o)DO-SIVS PF } \\
\text { AVG }\end{array}$ & $\mathrm{R} 1$ & GLORIA-ROMA(no)RVM & $(\mathrm{s}) \mathrm{MK}(-)$ & & 7 & 22 & 4.92 & $392-395$ & AE2 & $\mathrm{IX}, 27 \mathrm{a}$ \\
\hline 102 & \begin{tabular}{|c|} 
Ro-95-art \\
$141.526(33)$ \\
\end{tabular} & B1 & DN ARCADI-VS PF AVG & $\mathrm{R} 1$ & (gloria)-ROMANORVM & SMKB & & 1 & 21.5 & 5 & $392-395$ & AE2 & IX, 27b \\
\hline 103 & $\begin{array}{c}\text { Ro-95-art } \\
142.799 \\
\end{array}$ & B1 & DN HONORIVS PF AVG & $\mathrm{R} 1$ & GLOR(ia)-ROMANORVM & $\operatorname{SMK}(-)$ & & 6 & 21.5 & 4.81 & $392-395$ & AE2 & IX, 28c \\
\hline 104 & \begin{tabular}{|c|} 
Ro-97-art \\
$143.379(5)$ \\
\end{tabular} & B1 & $\begin{array}{c}\text { DN TH(eodo)-(siv)S PF } \\
\text { AVG }\end{array}$ & $\mathrm{R} 1$ & $\begin{array}{c}\text { GL(or)I(a)-(ro)MA(no) } \\
\text { RVM }\end{array}$ & SMKA & & 11 & 24 & 3.93 & $392-395$ & AE2 & IX, 27a \\
\hline 105 & \begin{tabular}{|c|} 
Ro-97-art \\
$143.379(33)$ \\
\end{tabular} & B1 & DN ARCADI-VS P(f avg) & $\mathrm{R} 1$ & GLO(ria)-ROM(an)ORVM & SMKГ & & 1 & 22.5 & 6.56 & $392-395$ & AE2 & IX, 27b \\
\hline 106 & \begin{tabular}{|c|} 
Ro-97-art \\
$143.379(60)$
\end{tabular} & $\mathrm{B} 1$ & DN HONORI(vs pf avg) & $\mathrm{R} 1$ & GLORIA-(romanorvm) & SMKГ & & 6 & 21.7 & 5.01 & $392-395$ & AE2 & $\mathrm{IX}, 28 \mathrm{c}$ \\
\hline
\end{tabular}

\begin{tabular}{|c|c|c|c|c|c|c|c|c|c|c|c|c|c|}
\hline \multirow[b]{2}{*}{$\mathrm{N}-1$} & \multicolumn{13}{|c|}{ HERACLEA } \\
\hline & N.R.A & A.T. & LEYENDA DE ANVERSO & R.T. & LEYENDA DE REVERSO & EXERGO & CAMPO & $\mathrm{C}$ & $\mathrm{M}$ & $P$ & CRON. & VALOR & R.I.C. \\
\hline 107 & \begin{tabular}{|c|} 
Ro-95-art \\
$141.415(1)$ \\
\end{tabular} & B1 & $\begin{array}{c}\text { DN THE(od)O-(sivs p) } \\
\text { F AVG }\end{array}$ & R1 & GLORIA-ROMANORVM & $(\mathrm{s}) \mathrm{MH}(-)$ & & 11 & 21.5 & 3.99 & $392-395$ & AE2 & IX, 27a \\
\hline 108 & $\begin{array}{c}\text { Ro-95-art } \\
141.415(27)\end{array}$ & B1 & $\begin{array}{c}\text { DN THE(odo)-SIVS PF } \\
\text { AVG }\end{array}$ & R1 & GLORIA-ROMANORVM & SMHA & & 6 & 21.5 & 4.25 & $392-395$ & AE2 & IX, 27a \\
\hline 109 & $\begin{array}{c}\text { Ro-95-art } \\
141.415(33)\end{array}$ & B1 & $\begin{array}{c}\text { DN THEO(do)-SIVS PF } \\
\text { AVG }\end{array}$ & $\mathrm{R} 1$ & $\begin{array}{c}\text { GLORIA- } \\
\text { ROMANORV }(\mathrm{m})\end{array}$ & $\mathrm{S}(\mathrm{m}) \mathrm{H}(-)$ & |* & 6 & 21.5 & 4.29 & $392-395$ & AE2 & IX, 27a \\
\hline 110 & \begin{tabular}{|c|} 
Ro-95-art \\
$141.481(1)$
\end{tabular} & B1 & (d)N HONORIVS P(f avg) & $\mathrm{R} 1$ & $\begin{array}{l}\text { (g)LORI(a)-(r) } \\
\text { OMANORVM }\end{array}$ & SMHA & & 11 & 21.6 & 6.71 & $392-395$ & AE2 & IX, 27c \\
\hline 111 & $\begin{array}{c}\text { Ro-95-art } \\
141.481(2)\end{array}$ & B1 & DN ARCADI-VS PF AVG & R1 & GLORIA-(roma)N(o)RVM & SMHA & |* & 12 & 22.2 & 5.50 & $392-395$ & AE2 & IX, 27b \\
\hline
\end{tabular}

2. Variante: solo presenta una cruz en campo.

3. Variante: solo presenta una cruz en campo. 


\begin{tabular}{|c|c|c|c|c|c|c|c|c|c|c|c|c|c|}
\hline 112 & $\begin{array}{c}\text { Ro-95-art } \\
141.509\end{array}$ & B1 & $\begin{array}{c}\text { DN THEODO-(siv)S PF } \\
\text { AV(g) }\end{array}$ & R1 & (glo)RIA-ROMANORVM & (s)MHB & I* $^{*}$ & 6 & 21.7 & 4.70 & $392-395$ & AE2 & IX, 27a \\
\hline 113 & $\begin{array}{c}\text { Ro-95-art } \\
141.526(11)\end{array}$ & B1 & DN ARCADI-(vs pf avg) & R1 & (gloria)-(roma)NORVM & SMHB & & 11 & 23.2 & 4.53 & $392-395$ & AE2 & IX, 27b \\
\hline 114 & $\begin{array}{c}\text { Ro-95-art } \\
141.526(24)\end{array}$ & B1 & $\begin{array}{c}\text { DN THEODO-SIVS PF } \\
\text { AVG }\end{array}$ & R1 & GLORIA-(ro)MANORVM & SMHA & $I^{*}$ & 12 & 22 & 4.51 & $392-395$ & AE2 & IX, 27a \\
\hline 115 & $\begin{array}{c}\text { Ro-95-art } \\
142.214\end{array}$ & B1 & DN ARCADI-(vs pf avg) & R1 & $\begin{array}{c}\text { GL(o)RI(a)- } \\
\text { ROMANORVM }\end{array}$ & SMHA & $\left.\right|^{*}$ & 6 & 21.2 & 3.98 & $392-395$ & AE2 & IX, 27b \\
\hline 116 & $\begin{array}{c}\text { Ro-97-art } \\
143.379(37)\end{array}$ & B1 & DN ARCADI-(vs pf avg) & R1 & $\begin{array}{c}\text { GLORIA-(r) } \\
\text { OMANOR(vm) }\end{array}$ & SMHA & & 11 & 21.8 & 5.48 & $392-395$ & AE2 & IX, 27b \\
\hline
\end{tabular}

\begin{tabular}{|c|c|c|c|c|c|c|c|c|c|c|c|c|c|}
\hline & \multicolumn{13}{|c|}{ LUGDUNUM } \\
\hline $\mathrm{N}-1$ & N.R.A & A.T. & LEYENDA DE ANVERSO & R.T. & LEYENDA DE REVERSO & EXERGO & CAMPO & $\mathrm{C}$ & $\mathrm{M}$ & $\mathrm{P}$ & CRON. & VALOR & R.I.C. \\
\hline 117 & $\begin{array}{c}\text { Ro-95-art } \\
141.389\end{array}$ & $\mathrm{~B} 1$ & DN GRATI(a)-(nvs pf avg) & $\mathrm{R} 2$ & REPARA(tio)-(reipv)B & LVGS & & 7 & 24.5 & 4.37 & $378-383$ & AE2 & IX, 28a \\
\hline 118 & $\begin{array}{c}\text { Ro-95-art } \\
141.458(1)\end{array}$ & B1 & (dn gratia)-N(vs pf) AVG & $\mathrm{R} 2$ & (reparatio)-(r)EIPVB & LVGS & | S & 12 & 22 & 4.63 & 378-383 & AE2 & $\mathrm{IX}, 28 \mathrm{a}$ \\
\hline 119 & $\begin{array}{c}\text { Ro-95-art } \\
141.526(14) \\
\end{array}$ & B1 & $\begin{array}{c}\text { DN (mag maxi)-MVS (pf } \\
\text { avg) }\end{array}$ & $\mathrm{R} 2$ & (repa)RATIO-REIPVB & LVGS & & 6 & 21.9 & 5.13 & $383-388$ & AE2 & IX, 32 \\
\hline 120 & \begin{tabular}{|c|} 
Ro-97-art \\
$143.379(22)$
\end{tabular} & B1 & (dn gratia)-NVS PF AVG & $\mathrm{R} 2$ & (reparati)O-RE(ip)V(b) & (1)VGP & & 12 & 22 & 3.85 & $378-383$ & $\mathrm{AE} 2$ & $\mathrm{IX}, 28 \mathrm{a}$ \\
\hline 121 & \begin{tabular}{|c|} 
Ro-97-art \\
$143.379(26)$
\end{tabular} & B1 & $\begin{array}{c}\text { DN VA(lentinianvs iv)N } \\
\text { PF AVG } \\
\end{array}$ & R2 & REPARA(tio)-(reipvb) & LVGP & |S & 6 & 23.1 & 5.35 & $378-383$ & AE2 & $\mathrm{IX}, 28 \mathrm{~b}$ \\
\hline 122 & \begin{tabular}{|c|} 
Ro-97-art \\
$143.379(27)$
\end{tabular} & B2 & $\begin{array}{l}\text { DN DECENTIVS NOB } \\
\text { (caes) }\end{array}$ & R3 & $\begin{array}{c}\text { VICTO(riae dd nn avg) ET } \\
\text { CAES }\end{array}$ & RSLC & SV & 7 & 21 & 5.46 & $350-353$ & $\mathrm{AE} 3$ & VIII, 124 \\
\hline
\end{tabular}

\begin{tabular}{|c|c|c|c|c|c|c|c|c|c|c|c|c|c|}
\hline & \multicolumn{13}{|c|}{ NICOMEDIA } \\
\hline $\mathrm{N}-1$ & N.R.A & A.T. & LEYENDA DE ANVERSO & R.T. & LEYENDA DE REVERSO & EXERGO & CAMPO & $\mathrm{C}$ & M & $P$ & CRON. & VALOR & R.I.C. \\
\hline 123 & \begin{tabular}{|c|} 
Ro-95-art \\
$141.387(1)$ \\
\end{tabular} & B1 & DN HONO(ri)VS PF (avg) & $\mathrm{R} 1$ & $\begin{array}{l}\text { GLORIA-ROM(a) } \\
\text { NORV(m) }\end{array}$ & $\operatorname{SMN}(-)$ & & 1 & 21.9 & 4.68 & $392-395$ & $\mathrm{AE} 2$ & IX, 46c \\
\hline 124 & \begin{tabular}{|c|} 
Ro-95-art \\
$141.387(3)$ \\
\end{tabular} & B1 & $\begin{array}{c}\text { (d)N VA(len)TINI-ANVS } \\
\text { PF AV(g) }\end{array}$ & R7 & (virtvs e)-XERCITI & (s)MNB & & 7 & 24 & 4.26 & $383-388$ & $\mathrm{AE} 2$ & IX, 44a \\
\hline 125 & \begin{tabular}{|c|} 
Ro-95-art \\
$141.390(2)$ \\
\end{tabular} & B1 & $\mid$ DN ARCADI-VS PF AVG & $\mathrm{R} 1$ & (gl)ORIA-RO(manorvm) & $\mathrm{SM}(\mathrm{n}) \mathrm{A}$ & & 11 & 21 & 5.31 & $392-395$ & AE2 & IX, 46b \\
\hline 126 & \begin{tabular}{|c|} 
Ro-95-art \\
$141.390(10)$ \\
\end{tabular} & B1 & DN HON(ori)VS PF AVG & $\mathrm{R} 1$ & GLORIA-ROMANORVM & SMNГ & & 6 & 23 & 4.39 & $392-395$ & $\mathrm{AE} 2$ & IX, $46 \mathrm{c}$ \\
\hline 127 & \begin{tabular}{|c|} 
Ro-95-art \\
$141.390(22)$ \\
\end{tabular} & B1 & (dn h)O(no)RIVS (pf avg) & $\mathrm{R} 1$ & GLORIA-(roman)ORVM & $(-) \mathrm{MN} \Gamma$ & & 6 & 20 & 3.73 & $392-395$ & AE2 & IX, $46 \mathrm{c}$ \\
\hline 128 & \begin{tabular}{|c|} 
Ro-95-art \\
$141.415(15)$ \\
\end{tabular} & B1 & $(\mathrm{dn} h)$ ONORIVS PF AV(g) & R1 & (gloria)-ROMANORVM & SMNГ & & 7 & 22 & 4.78 & $392-395$ & $\mathrm{AE} 2$ & IX, 46c \\
\hline 129 & \begin{tabular}{|c|} 
Ro-95-art \\
$141.415(39)$ \\
\end{tabular} & B1 & DN HONORIVS (pf avg) & $\mathrm{R} 1$ & GLORIA-ROM(anor)VM & SMNГ & & 6 & 20 & 2.96 & $392-395$ & $\mathrm{AE} 2$ & IX, 46c \\
\hline 130 & \begin{tabular}{|c|} 
Ro-95-art \\
$141.466(3)$ \\
\end{tabular} & B1 & $\begin{array}{c}\text { DN THE(odo)-SI(vs) PF } \\
\text { AVG } \\
\end{array}$ & $\mathrm{R} 7$ & (virtvs e)-(x)ERC(it)I & (s)MN(-) & & 6 & 22.5 & 4.31 & $383-388$ & $\mathrm{AE} 2$ & IX, 44b \\
\hline 131 & \begin{tabular}{|c|} 
Ro-95-art \\
$141.481(3)$ \\
\end{tabular} & B1 & DN HONOR(ivs p)F A(vg) & $\mathrm{R} 1$ & $\begin{array}{l}\text { (g)LORIA-(r) } \\
\text { OMANORVM }\end{array}$ & SMNГ & & 7 & 21.9 & 4.42 & $392-395$ & AE2 & IX, 46c \\
\hline 132 & $\begin{array}{c}\text { Ro-95-art } \\
141.495 \\
\end{array}$ & B1 & $\begin{array}{c}\text { DN VALEN(tini)ANVS } \\
\text { PF AVG } \\
\end{array}$ & R2 & REPAR(atio)-(re)IPVB & SMNB & & 11 & 24 & 4.53 & $378-383$ & AE2 & IX, 27b \\
\hline 133 & \begin{tabular}{|c|} 
Ro-95-art \\
$141.526(4)$ \\
\end{tabular} & B1 & (dn) ARCADI-(vs pf avg) & R1 & GLORIA-(romanorvm) & $\mathrm{SM}(\mathrm{n}-)$ & & 6 & 20 & 4.81 & $392-395$ & AE2 & $\mathrm{IX}, 46 \mathrm{~b}$ \\
\hline 134 & \begin{tabular}{|c|} 
Ro-95-art \\
$141.526(7)$ \\
\end{tabular} & B1 & (dn arc)ADI-VS P(f avg) & R1 & (gloria-romanorvm) & SMNB & & 6 & 20.5 & 5.30 & $392-395$ & AE2 & IX, 46b \\
\hline 135 & \begin{tabular}{|c|} 
Ro-95-art \\
$141.526(16)$ \\
\end{tabular} & B1 & DN AR(cadi)-VS PF AVG & $\mathrm{R} 1$ & GLORIA-(romano)RVM & SMNB & & 12 & 22 & 4.34 & $392-395$ & $\mathrm{AE} 2$ & IX, 46b \\
\hline 136 & \begin{tabular}{|c|} 
Ro-95-art \\
$141.526(25)$ \\
\end{tabular} & B1 & $\begin{array}{c}\text { DN TH(eodo)-(si)VS P(f } \\
\text { avg) }\end{array}$ & R2 & (re)PARATIO-(reipvb) & (s)MNB & & 5 & 22.7 & 3.19 & $378-383$ & $\mathrm{AE} 2$ & IX, 27a \\
\hline 137 & \begin{tabular}{|c|} 
Ro-95-art \\
$141.526(27)$ \\
\end{tabular} & B1 & (dn th)EODO-(sivs pf avg) & $\mathrm{R} 1$ & (gloria)-(roman)ORVM & SMNA & & 12 & 21.1 & 4.10 & $392-395$ & AE2 & IX, 46a \\
\hline 138 & \begin{tabular}{|c|} 
Ro-95-art \\
$141.526(34)$ \\
\end{tabular} & B1 & $\mathrm{DN}(\operatorname{arc}) \mathrm{ADI}-(\mathrm{vs} p \mathrm{pf}$ a)VG & $\mathrm{R} 1$ & GLORIA-(romano)RVM & SMNB & & 6 & 22.2 & 4.90 & $392-395$ & $\mathrm{AE} 2$ & IX, 46b \\
\hline 139 & \begin{tabular}{|c|} 
Ro-95-art \\
142.795 \\
\end{tabular} & B1 & & $\mathrm{R} 1$ & (gloria)-(r)OMANORVM & SMNA & & 6 & 21 & 4.84 & & & \\
\hline 140 & $\begin{array}{l}\text { Ro-95-art } \\
142.802\end{array}$ & B1 & DN ARCADI-VS PF AVG & $\mathrm{R} 1$ & GLORIA-ROMANORVM & SMNB & & 7 & 22 & 4.47 & $392-395$ & AE2 & IX, 46b \\
\hline 141 & $\begin{array}{c}\text { Ro-95-art } \\
142.853 \\
\end{array}$ & B1 & (dn gr)ATIA-NV(s pf avg) & R2 & REPARATIO-(reipvb) & $\operatorname{SMN}(-)$ & & 6 & 21 & 5.33 & $378-383$ & AE2 & IX, 27a \\
\hline 142 & \begin{tabular}{|c|} 
Ro-97-art \\
$143.379(18)$ \\
\end{tabular} & B1 & $\begin{array}{c}\text { (dn the)O(do)-SIVS PF } \\
\mathrm{A}(\mathrm{vg})\end{array}$ & $\mathrm{R} 1$ & G(1)ORI(a)-RO(manorv)M & SMNA & & 6 & 21.7 & 4.12 & $392-395$ & $\mathrm{AE} 2$ & IX, 46a \\
\hline 143 & \begin{tabular}{|c|} 
Ro-97-art \\
$143.379(28)$ \\
\end{tabular} & B1 & (dn gratia)-NVS PF AVG & R2 & (repara)TIO-(reipvb) & $\operatorname{SMN}(-)$ & & 12 & 24 & 3.97 & $378-383$ & $\mathrm{AE} 2$ & IX, 27a \\
\hline 144 & \begin{tabular}{|c|} 
Ro-97-art \\
$143.379(38)$ \\
\end{tabular} & B1 & DN ARCADI-VS PF AVG & R1 & $\begin{array}{l}\mathrm{G}(\mathrm{l}) \mathrm{O} \text { (ria)-ROM(an) } \\
\text { ORVM }\end{array}$ & SMNA & & 6 & 20 & 5.28 & $392-395$ & $\mathrm{AE} 2$ & IX, 46b \\
\hline
\end{tabular}




\begin{tabular}{|c|c|c|c|c|c|c|c|c|c|c|c|c|}
\hline 145 & \begin{tabular}{|c|} 
Ro-97-art \\
$143.379(39)$ \\
\end{tabular} & B3 & $\begin{array}{c}\text { DN ARCAD(i)-(v)S PF } \\
\text { AVG }\end{array}$ & $\mathrm{R} 4$ & (glo)RIA RO-MANORVM & (s) $\mathrm{MN}(-)$ & 6 & 22.9 & 4.55 & $378-383$ & AE2 & IX, 26 \\
\hline 146 & \begin{tabular}{|c|} 
Ro-97-art \\
$143.379(40)$ \\
\end{tabular} & B1 & $\begin{array}{l}\text { (d)N AR(c)ADI-VS (pf } \\
\text { avg) }\end{array}$ & $\mathrm{R} 1$ & GLORIA-RO(manorvm) & SMNB & 5 & 22 & 4.47 & $392-395$ & AE2 & IX, 46b \\
\hline 147 & \begin{tabular}{|c|} 
Ro-97-art \\
$143.379(41)$ \\
\end{tabular} & B1 & DN ARCADI-VS (pf av)G & $\mathrm{R} 1$ & (gl)ORIA-(romanorvm) & $\operatorname{SMN}(-)$ & 6 & 22 & 4.72 & $392-395$ & AE2 & IX, 46b \\
\hline 148 & \begin{tabular}{|c|} 
Ro-97-art \\
$143.379(42)$ \\
\end{tabular} & B1 & DN ARCADI-(vs pf avg) & $\mathrm{R} 1$ & $\begin{array}{l}\text { GLORIA-(r)O(ma) } \\
\text { NORVM }\end{array}$ & SMNB & 12 & 22 & 3.68 & $392-395$ & AE2 & IX, 46b \\
\hline 149 & \begin{tabular}{|c|} 
Ro-97-art \\
$143.379(45)$ \\
\end{tabular} & B1 & DN GRAT(ia)-(nvs pf avg) & $\mathrm{R} 2$ & (reparatio-reipvb) & $\mathrm{SM}(\mathrm{n}) \mathrm{B}$ & 11 & 25.8 & 6.35 & $378-383$ & AE2 & IX, 27a \\
\hline 150 & \begin{tabular}{|c|} 
Ro-97-art \\
$143.379(59)$
\end{tabular} & B1 & (dn ho)NORIVS (pf avg) & $\mathrm{R} 1$ & (gloria)-ROMA(norvm) & SMNГ & 11 & 20 & 5.03 & $392-395$ & AE2 & IX, 46c \\
\hline 151 & \begin{tabular}{|c|} 
Ro-97-art \\
$143.379(63)$
\end{tabular} & B1 & $\mathrm{DN} \mathrm{HO}(\mathrm{n}) \mathrm{O}(\mathrm{ri}) \mathrm{V}(\mathrm{s}$ pf avg) & $\mathrm{R} 1$ & (gloria)-(ro)MANORVM & SMNГ & 6 & 21 & 3.93 & $392-395$ & AE2 & IX, 46c \\
\hline
\end{tabular}

\begin{tabular}{|c|c|c|c|c|c|c|c|c|c|c|c|c|c|}
\hline \multirow[b]{2}{*}{$\mathrm{N}-1$} & \multicolumn{13}{|c|}{ ROMA } \\
\hline & N.R.A & A.T. & LEYENDA DE ANVERSO & R.T. & LEYENDA DE REVERSO & EXERGO & CAMPO & $\mathrm{C}$ & $\mathrm{M}$ & $P$ & CRON. & VALOR & R.I.C. \\
\hline 152 & $\begin{array}{l}\text { Ro-95-art } \\
141.388\end{array}$ & B1 & (dn theo)DO-(sivs pf avg) & $\mathrm{R} 2$ & REPARATIO-REIPVB & (s)MR(-) & & 12 & 23.8 & 2.97 & $378-383$ & AE2 & IX, 43d \\
\hline 153 & \begin{tabular}{|c|} 
Ro-95-art \\
$141.390(11)$ \\
\end{tabular} & B1 & $\begin{array}{l}\text { DN GRATIA-NVS PF } \\
\text { (avg) }\end{array}$ & $\mathrm{R} 2$ & REPARATIO-R(eipv)B & SMRT & & 12 & 23 & 4.63 & $378-383$ & AE2 & IX, 43a \\
\hline 154 & \begin{tabular}{|c|} 
Ro-95-art \\
$141.415(4)$ \\
\end{tabular} & B1 & $\begin{array}{l}\text { DN THEO(do)-(sivs pf } \\
\text { avg) }\end{array}$ & $\mathrm{R} 2$ & (reparat)IO-REIPVB & SMRQ & & 11 & 24.5 & 5.02 & $378-383$ & AE2 & IX, 43d \\
\hline 155 & \begin{tabular}{|l|} 
Ro-95-art \\
$141.415(6)$
\end{tabular} & B1 & $\begin{array}{c}\text { DN THEODO-(sivs pf) } \\
\text { AVG }\end{array}$ & $\mathrm{R} 2$ & REPA(ra)TIO-REIP(vb) & (s)MR(-) & & 6 & 23.5 & 5.35 & $378-383$ & AE2 & IX, 43d \\
\hline 156 & \begin{tabular}{|c|} 
Ro-95-art \\
$141.415(22)$ \\
\end{tabular} & B6 & $\begin{array}{l}\text { IMP MAXIMI(nvs) PF } \\
\text { (avg) }\end{array}$ & R9 & SOL I(inv)-IC-TO COMITI & RS & & 1 & 21.8 & 4.16 & $312-313$ & AEFollis & VI, 335b \\
\hline 157 & $\begin{array}{c}\text { Ro-95-art } \\
141.526(8)\end{array}$ & B1 & DN TH(eodo)-(sivs pf avg) & $\mathrm{R} 2$ & (repar)ATIO-(reipvb) & $\mathrm{SM}(\mathrm{r}) \mathrm{P}$ & & 12 & 24 & 4.05 & $378-383$ & AE2 & IX, 43d \\
\hline 158 & $\begin{array}{c}\text { Ro-95-art } \\
141.458(5)\end{array}$ & B1 & $\begin{array}{l}\text { DN (g)RATIA-NVS (pf) } \\
\text { AV }(\mathrm{g})\end{array}$ & $\mathrm{R} 2$ & (reparat)IO-REIPVB & SMRT & & 12 & 23 & 6.27 & $378-383$ & AE2 & IX, 43a \\
\hline 159 & \begin{tabular}{|c|} 
Ro-95-art \\
$142.215(1)$ \\
\end{tabular} & B1 & $\begin{array}{l}\text { DN GRA(tia)-NVS (pf } \\
\text { avg) }\end{array}$ & $\mathrm{R} 2$ & REPA(ratio)-(rei)PVB & SMRT & & 12 & 23.1 & 4.92 & $378-383$ & AE2 & IX, 43a \\
\hline 160 & \begin{tabular}{|c|} 
Ro-97-art \\
$143.379(25)$ \\
\end{tabular} & B1 & $\begin{array}{l}\text { (dn) VALENTINIANVS } \\
\text { (pf) AVG }\end{array}$ & $\mathrm{R} 2$ & (re)PAR(at)IO-(rei)PVB & SMRT & & 12 & 23.5 & 5.01 & $378-383$ & AE2 & IX, $43 \mathrm{c}$ \\
\hline 161 & \begin{tabular}{|c|} 
Ro-97-art \\
$143.379(51)$ \\
\end{tabular} & B1 & DN GRATIA-(nvs pf avg) & $\mathrm{R} 2$ & (repa)RATI(o)-(reipvb) & SMRQ & & 6 & 23 & 5.50 & $378-383$ & AE2 & $\mathrm{IX}, 43 \mathrm{a}$ \\
\hline 162 & \begin{tabular}{|c|} 
Ro-97-art \\
$143.379(53)$ \\
\end{tabular} & B1 & $\begin{array}{c}\text { DN GRATIA-N(vs) PF } \\
\text { A(vg) }\end{array}$ & $\mathrm{R} 2$ & (r)EPARA(tio)-(reipv)B & SMRQ & & 5 & 23.1 & 4.29 & $378-383$ & AE2 & IX, 43a \\
\hline 163 & \begin{tabular}{|c|} 
Ro-97-art \\
$143.379(68)$ \\
\end{tabular} & B1 & $\begin{array}{c}\text { (dn) MAG MA-(ximv)S } \\
\text { PF A(vg) }\end{array}$ & $\mathrm{R} 2$ & (reparatio-reipvb) & SMR & & 6 & 24 & 5.36 & $383-388$ & AE2 & IX, 54 \\
\hline
\end{tabular}

\begin{tabular}{|c|c|c|c|c|c|c|c|c|c|c|c|c|c|}
\hline \multirow[b]{2}{*}{$\mathrm{N}-1$} & \multicolumn{13}{|c|}{ SISCIA } \\
\hline & N.R.A & A.T. & LEYENDA DE ANVERSO & R.T. & LEYENDA DE REVERSO & EXERGO & CAMPO & $\mathrm{C}$ & $\mathrm{M}$ & $\mathrm{P}$ & CRON. & VALOR & R.I.C. \\
\hline 164 & \begin{tabular}{|c|} 
Ro-95-art \\
$141.390(23)$ \\
\end{tabular} & $\mathrm{B} 1$ & $\begin{array}{c}\text { DN GRA(ti)A-NVS P(f) } \\
\text { AVG }\end{array}$ & $\mathrm{R} 2$ & REPARATIO-REIPVB & *BSISC & & 7 & 23.2 & 4.77 & $378-383$ & AE2 & IX, 26a \\
\hline 165 & \begin{tabular}{|c|} 
Ro-95-art \\
$141.397(1)$ \\
\end{tabular} & & & $\mathrm{R} 1$ & (gl)ORIA-(rom)ANORVM & $(-\mathrm{si}) \mathrm{SC}$ & & & 21.8 & 4.25 & & & \\
\hline 166 & \begin{tabular}{|c|} 
Ro-95-art \\
$141.526(31)$ \\
\end{tabular} & $\mathrm{B} 1$ & $\begin{array}{c}\text { DN THEODO-SIVS PF } \\
\mathrm{A}(\mathrm{vg})\end{array}$ & $\mathrm{R} 2$ & REPARATIO-REIPVB & $*$ BSISC $\bullet$ & & 12 & 24 & 5.59 & $378-383$ & AE2 & IX, 26c \\
\hline 167 & $\begin{array}{c}\text { Ro-95-art } \\
142.842\end{array}$ & $\mathrm{~B} 1$ & & $\mathrm{R} 1$ & GL(oria)-(roman)ORVM & $(-) \mathrm{S}($ is $) \mathrm{C}$ & & 12 & 21 & 4.06 & & & \\
\hline 168 & \begin{tabular}{|c|} 
Ro-97-art \\
$143.379(47)$
\end{tabular} & B1 & $\begin{array}{c}\text { DN GRATIA-NVS PF } \\
\text { AVG }\end{array}$ & $\mathrm{R} 2$ & (re)PAR(atio)-REIPVB & ASISC• & & 1 & 23.1 & 4.60 & $378-383$ & AE2 & IX, 26a \\
\hline
\end{tabular}

\begin{tabular}{|c|c|c|c|c|c|c|c|c|c|c|c|c|c|}
\hline & \multicolumn{10}{|c|}{ TESALÓNICA } \\
\hline N-1 & N.R.A & A.T. & LEYENDA DE ANVERSO & R.T. & LEYENDA DE REVERSO & EXERGO & CAMPO & C & M & P & CRON. & VALOR & R.I.C. \\
\hline 169 & $\begin{array}{c}\text { Ro-95-art } \\
141.458(4)\end{array}$ & B1 & $\begin{array}{c}\text { (dn the)ODO-(s)IV(s pf } \\
\text { avg) }\end{array}$ & R2 & REPARA(tio)-REIPVB & TES & $\mid \Gamma$ & 5 & 23 & 4.36 & $378-383$ & AE2 & IX, 37d \\
\hline 170 & $\begin{array}{c}\text { Ro-97-art } \\
143.379(23)\end{array}$ & B1 & $\begin{array}{c}\text { DN VA(LENTI)NIANVS } \\
\text { PF A(vg) }\end{array}$ & R2 & (repa)RATIO-REIPVB & SMTES & $\mid \Delta$ & 6 & 23.2 & 4.98 & $378-383$ & AE2 & IX, 37b \\
\hline
\end{tabular}

\begin{tabular}{|c|c|c|c|c|c|c|c|c|c|c|c|c|c|}
\hline & \multicolumn{13}{|c|}{ TRÉVERIS } \\
\hline $\mathrm{N}-1$ & N.R.A & A.T. & LEYENDA DE ANVERSO & R.T. & LEYENDA DE REVERSO & EXERGO & CAMPO & $\mathrm{C}$ & $\mathrm{M}$ & $\mathrm{P}$ & \begin{tabular}{|l|} 
CRON. \\
\end{tabular} & VALOR & R.I.C. \\
\hline 171 & \begin{tabular}{|c|} 
Ro-97-art \\
$143.379(50)$
\end{tabular} & B1 & $\begin{array}{c}\text { (dn gr)ATIA-NVS (pf) } \\
\text { AVG }\end{array}$ & R2 & REPARATIO-RE(ipvb) & $\mathrm{SM}(\mathrm{tr}) \mathrm{S}$ & & 11 & 22.1 & 4.86 & $378-383$ & $\mathrm{AE} 2$ & IX, 65a \\
\hline
\end{tabular}

\begin{tabular}{|c|c|c|c|c|c|c|c|c|c|c|c|}
\hline & \multicolumn{11}{|c|}{ MONEDAS NO IDENTIFICADAS } \\
\hline $\mathrm{N}-1$ & N.R.A & A.T. LEYENDA DE ANVERSO & R.T. & \begin{tabular}{|l|l|} 
LEYENDA DE REVERSO & EXERGO \\
\end{tabular} & CAMPO & $\mathrm{C}$ & $\mathrm{M}$ & $P$ & CRON. & VALOR & R.I.C. \\
\hline
\end{tabular}




\begin{tabular}{|c|c|c|c|c|c|c|c|c|c|c|c|c|}
\hline 172 & $\begin{array}{l}\text { Ro-95-art } \\
141.384\end{array}$ & B1 & (dn g)RA(tia)-(nvs pf avg) & $\mathrm{R} 2$ & (repar)ATIO-(reipvb) & & & 11 & 21 & 4.19 & & \\
\hline 173 & \begin{tabular}{c|} 
Ro-95-art \\
$141.390(1)$
\end{tabular} & B1 & (dn arca)DIV(s pf avg) & R7 & (gl)ORIA-ROMANORVM & & & 1 & 21.2 & 4.23 & & \\
\hline 174 & \begin{tabular}{|c|} 
Ro-95-art \\
$141.390(3)$ \\
\end{tabular} & B1 & $\begin{array}{l}\text { (dn) GR(a)T(ia)-(nvs pf } \\
\text { avg) }\end{array}$ & $\mathrm{R} 2$ & (r)EPARATIO-REIP(vb) & & & 11 & 22.5 & 4.71 & & \\
\hline 175 & \begin{tabular}{|c|} 
Ro-95-art \\
$141.390(8)$ \\
\end{tabular} & $\mathrm{B} 1$ & $\begin{array}{c}\text { (dn valentini)-ANVS PF } \\
\text { AVG }\end{array}$ & $\mathrm{R} 2$ & RE(par)AT(io)-(reipvb) & & & 1 & 21.5 & 2.97 & & \\
\hline 176 & \begin{tabular}{|c|} 
Ro-95-art \\
$141.390(12)$ \\
\end{tabular} & $\mathrm{B} 1$ & $\begin{array}{c}\begin{array}{c}\text { DN MAG MAX(i)-(mvs } \\
\text { pf avg) }\end{array} \\
\end{array}$ & $\mathrm{R} 2$ & REPA(rati)O-(reipvb) & & & 7 & 25 & 4.05 & & \\
\hline 177 & \begin{tabular}{|c|} 
Ro-95-art \\
$141.390(13)$ \\
\end{tabular} & B1 & & & & & & & 22 & 5.19 & & \\
\hline 178 & \begin{tabular}{|c|} 
Ro-95-art \\
$141.390(19)$ \\
\end{tabular} & B1 & DN THEODO-(sivs pf avg) & R1 & (gl)ORIA-(romanorvm) & & & 12 & 21 & 4.09 & & \\
\hline 179 & \begin{tabular}{|c|} 
Ro-95-art \\
$141.390(20)$
\end{tabular} & B1 & DN GR(atia)-(nvs) PF AVG & $\mathrm{R} 2$ & (repara)TIO-R(eipvb) & & | S & 5 & 23.1 & 4.26 & & \\
\hline 180 & \begin{tabular}{|c|} 
Ro-95-art \\
$141.390(21)$ \\
\end{tabular} & $\mathrm{B} 1$ & (dn h)ONO(rivs pf avg) & $\mathrm{R} 1$ & (gl)ORIA-RO(manor)VM & & & 11 & 21.8 & 4.94 & & \\
\hline 181 & \begin{tabular}{|c|} 
Ro-95-art \\
$141.390(24)$ \\
\end{tabular} & $\mathrm{B} 1$ & & R1 & (gloria-romanorvm) & & & 12 & 22.2 & 4.19 & & \\
\hline 182 & \begin{tabular}{|c|} 
Ro-95-art \\
$141.390(26)$ \\
\end{tabular} & B1 & $\begin{array}{l}\text { DN MAG MAXI-(mvs } \\
\text { pf avg) }\end{array}$ & & & & & & 22.6 & 4.19 & & \\
\hline 183 & \begin{tabular}{c|} 
Ro-95-art \\
141.395 \\
\end{tabular} & & & & & & & & 21.5 & 3.27 & & \\
\hline 184 & $\begin{array}{l}\text { Ro-95-art } \\
141.396\end{array}$ & $\mathrm{~B} 1$ & (dn gr)AT(ia)-NVS (pf avg) & $\mathrm{R} 2$ & (r)EPAR(atio)-(reipv)B & $(-) \mathrm{N}$ & & 6 & 24 & 5.55 & & \\
\hline 185 & \begin{tabular}{|c|} 
Ro-95-art \\
$141.397(2)$ \\
\end{tabular} & $\mathrm{B} 1$ & $\begin{array}{c}\text { DN MA(g maxi)-(m)VS } \\
\text { (pf) AVG }\end{array}$ & $\mathrm{R} 2$ & (reparatio)-REIPVB & & & 6 & 23 & 3.75 & & \\
\hline 186 & \begin{tabular}{|c|} 
Ro-95-art \\
$141.415(7)$ \\
\end{tabular} & B1 & $\begin{array}{l}\text { DN VALENTINIANVS } \\
\text { PF AVG }\end{array}$ & $\mathrm{R} 2$ & (r)EPARATIO-REIPVB & & & 11 & 25.5 & 3.71 & & \\
\hline 187 & \begin{tabular}{|c|} 
Ro-95-art \\
$141.415(10)$ \\
\end{tabular} & B1 & $\begin{array}{l}\text { DN MAG MAXI-MVS } \\
\text { (pf avg) }\end{array}$ & $\mathrm{R} 2$ & REPA(ratio)-REI(pvb) & & & 6 & 22.8 & 4.67 & & \\
\hline 188 & \begin{tabular}{|c|} 
Ro-95-art \\
$141.415(19)$
\end{tabular} & B1 & $\begin{array}{l}\text { (dn the)ODO-SIVS (pf } \\
\text { avg) }\end{array}$ & $\mathrm{R} 1$ & (gloria)-ROMAN(orvm) & & & 12 & 19 & 5.17 & & \\
\hline 189 & \begin{tabular}{|c|} 
Ro-95-art \\
$141.415(34)$ \\
\end{tabular} & B1 & $\begin{array}{c}\text { (dn gr)A(ti)-ANVS PF } \\
\text { (avg) }\end{array}$ & $\mathrm{R} 2$ & (reparatio)-(rei)PVB & $(-) \mathrm{A}$ & & 5 & 23 & 4.31 & & \\
\hline 190 & \begin{tabular}{|c|} 
Ro-95-art \\
$141.415(37)$ \\
\end{tabular} & B1 & $\begin{array}{c}\text { (dn) THEODO-SIVS P(f } \\
\text { avg) }\end{array}$ & R1 & $\begin{array}{c}\text { (g)LORIA- } \\
\text { ROMANO(rvm) }\end{array}$ & & & 1 & 20 & 4.50 & & \\
\hline 191 & \begin{tabular}{|c|} 
Ro-95-art \\
$141.452(4)$ \\
\end{tabular} & & & & & & & & 25 & 6.24 & & \\
\hline 192 & \begin{tabular}{|c|} 
Ro-95-art \\
$141.452(6)$ \\
\end{tabular} & B1 & $\begin{array}{c}\text { (dn c)ONSTA-NS PF } \\
\mathrm{AV}(\mathrm{g})\end{array}$ & R6 & (fel temp)-REPA(ratio) & & & 5 & 21.5 & 4.17 & & \\
\hline 193 & \begin{tabular}{|c|} 
Ro-95-art \\
$141.458(3)$ \\
\end{tabular} & & & & & & & & 22 & 4.87 & & \\
\hline 194 & \begin{tabular}{|c|} 
Ro-95-art \\
$141.466(1)$ \\
\end{tabular} & B1 & (...)VS PF AVG & R1 & (gloria)-ROMANOR(vm) & & & 6 & 20.5 & 6.21 & & \\
\hline 195 & \begin{tabular}{|c|} 
Ro-95-art \\
$141.466(2)$ \\
\end{tabular} & B1 & & & & & & & 21.5 & 4.49 & & \\
\hline 196 & \begin{tabular}{|c|} 
Ro-95-art \\
$141.466(4)$ \\
\end{tabular} & $\mathrm{B} 1$ & $\begin{array}{c}\begin{array}{c}\text { DN THEODO-(siv)S PF } \\
\text { AVG }\end{array} \\
\end{array}$ & R1 & G(lo)RIA-ROM(anorvm) & & & 9 & 21 & 4.49 & & \\
\hline 197 & \begin{tabular}{|c|} 
Ro-95-art \\
$141.487(1)$ \\
\end{tabular} & & & R1 & (gloria-romanorvm) & & & & 22.1 & 5.25 & & \\
\hline 198 & \begin{tabular}{|c|} 
Ro-95-art \\
$141.487(2)$
\end{tabular} & B1 & $\begin{array}{l}\text { DN VALENTINIA(nvs) } \\
\text { PF AVG }\end{array}$ & & & & & & 24 & 4.54 & & \\
\hline 199 & \begin{tabular}{|c|} 
Ro-95-art \\
$141.515(1)$ \\
\end{tabular} & B1 & (dn) HONORIVS P(f avg) & R1 & $\begin{array}{c}\text { (g)LOR(ia)-RO(ma)NO(r) } \\
\text { VM }\end{array}$ & & & 1 & 21.8 & 4.45 & & \\
\hline 200 & \begin{tabular}{|c|} 
Ro-95-art \\
$141.515(3)$ \\
\end{tabular} & B1 & $\begin{array}{c}\text { (d)N HONORIVS PF } \\
\mathrm{A}(\mathrm{vg})\end{array}$ & $\mathrm{R} 1$ & (gloria)-ROMANORVM & & & 4 & 20.5 & 4.25 & & \\
\hline 201 & \begin{tabular}{|c|} 
Ro-95-art \\
$141.515(4)$ \\
\end{tabular} & B1 & & $\mathrm{R} 2$ & (reparatio-reipvb) & & & 5 & 23 & 4.06 & & \\
\hline 202 & \begin{tabular}{|c|} 
Ro-95-art \\
$141.526(20)$ \\
\end{tabular} & B1 & & $\mathrm{R} 2$ & (repa)RATIO-(rei)PVB & & & 5 & 22.2 & 4.19 & & \\
\hline 203 & \begin{tabular}{|c|} 
Ro-95-art \\
$141.526(23)$ \\
\end{tabular} & B1 & & R2 & (repar)A(tio)-RE(ipvb) & & & 6 & 21.9 & 3.90 & & \\
\hline 204 & \begin{tabular}{|c|} 
Ro-95-art \\
$141.526(26)$ \\
\end{tabular} & $\mathrm{B} 1$ & $\begin{array}{l}\text { DN GRATIA-NVS PF } \\
\text { AVG }\end{array}$ & $\mathrm{R} 2$ & REPARA(tio)-(reipvb) & & & 6 & 24 & 3.66 & & \\
\hline 205 & \begin{tabular}{|c|} 
Ro-95-art \\
$141.526(28)$ \\
\end{tabular} & B1 & (d)N ARCADI-(vs pf avg) & & & & & & 21 & 4.09 & & \\
\hline 206 & \begin{tabular}{|c|} 
Ro-95-art \\
$141.526(30)$ \\
\end{tabular} & $\mathrm{B} 1$ & $\begin{array}{l}\text { (d)N GRATIA-NVS PF } \\
\text { AVG }\end{array}$ & $\mathrm{R} 2$ & (reparat)IO-(reipvb) & & & 5 & 22 & 4.79 & & \\
\hline 207 & \begin{tabular}{|c|} 
Ro-95-art \\
$141.526(32)$ \\
\end{tabular} & B1 & $\begin{array}{l}\text { DN TH(eodo)-(sivs pf) } \\
\text { AVG }\end{array}$ & $\mathrm{R} 2$ & (re)PARATIO-REIPVB & & & 12 & 23 & 4.99 & & \\
\hline 208 & \begin{tabular}{|c|} 
Ro-95-art \\
$141.526(35)$ \\
\end{tabular} & & & & & & & & 20.9 & 4.35 & & \\
\hline
\end{tabular}




\begin{tabular}{|c|c|c|c|c|c|c|c|c|c|c|c|c|c|}
\hline 209 & $\begin{array}{l}\text { Ro-95-art } \\
142.796\end{array}$ & B1 & (dn gratia)-NVS $\mathrm{P}(\mathrm{f}$ avg) & $\mathrm{R} 2$ & (reparatio)-(reip)VB & & & 6 & 21.7 & 4.59 & & & \\
\hline 210 & $\begin{array}{c}\text { Ro-95-art } \\
142.798 \\
\end{array}$ & B1 & $\begin{array}{c}\text { DN MAG M(axi)-(mvs } \\
\text { pf avg) }\end{array}$ & $\mathrm{R} 2$ & (reparatio-reipvb) & & & 1 & 23.2 & 4.82 & & & \\
\hline 211 & $\begin{array}{c}\text { Ro-95-art } \\
142.800\end{array}$ & B1 & DN ARCADIVS PF AVG & $\mathrm{R} 1$ & (gloria)-ROMANORVM & & & 5 & 21 & 4.12 & & & \\
\hline 212 & $\begin{array}{c}\text { Ro-95-art } \\
142.801\end{array}$ & B1 & & $\mathrm{R} 2$ & (reparatio-reipvb) & & & 6 & 22.3 & 4.34 & & & \\
\hline 213 & $\begin{array}{c}\text { Ro-95-art } \\
142.832\end{array}$ & B1 & $\begin{array}{c}\text { (dn) A(r)C(adi)V(s pf) } \\
\text { AVG }\end{array}$ & $\mathrm{R} 1$ & (glo)RIA-ROMANORVM & & & 6 & 21.2 & 4.32 & & & \\
\hline 214 & $\begin{array}{c}\text { Ro-95-art } \\
142.840\end{array}$ & B1 & $\begin{array}{c}\text { DN GR(ati)A-NVS PF } \\
\text { AVG }\end{array}$ & $\mathrm{R} 2$ & (reparatio-reipvb) & & & 11 & 22.5 & 3.85 & & & \\
\hline 215 & $\begin{array}{c}\text { Ro-95-art } \\
142.851\end{array}$ & & & R1 & GLOR(ia)-(romanorvm) & & & & 23.5 & 2.03 & & & \\
\hline 216 & $\begin{array}{c}\text { Ro-95-art } \\
142.855\end{array}$ & B1 & & $\mathrm{R} 2$ & (reparatio-reipvb) & & & 6 & 23.8 & 4.18 & & & \\
\hline 217 & $\begin{array}{c}\text { Ro-95-art } \\
143.054(3)\end{array}$ & B1 & & $\mathrm{R} 1$ & (gl)ORIA-ROMANORVM & & & 6 & 18.5 & 2.17 & & & \\
\hline 218 & \begin{tabular}{|c|} 
Ro-97-art \\
$143.379(1)$ \\
\end{tabular} & B1 & $\begin{array}{l}\text { DN THEODO-SIVS (pf) } \\
\text { AVG }\end{array}$ & & & & & & 21.5 & 4.26 & & & \\
\hline 219 & \begin{tabular}{|c|} 
Ro-97-art \\
$143.379(2)$ \\
\end{tabular} & B1 & $\begin{array}{c}\text { (dn t)HEODO-SIVS (pf } \\
\text { avg) }\end{array}$ & $\mathrm{R} 2$ & (rep)AR(atio)-(reipvb) & & & 6 & 22.5 & 4.27 & & & \\
\hline 220 & \begin{tabular}{|c|} 
Ro-97-art \\
$143.379(7)$
\end{tabular} & B1 & $\begin{array}{l}\text { DN THE(odo)-(si)VS PF } \\
\text { AVG }\end{array}$ & $\mathrm{R} 1$ & (glor)IA-(ro)MANORVM & & & 6 & 22 & 4.62 & & & \\
\hline 221 & \begin{tabular}{|c|} 
Ro-97-art \\
$143.379(11)$ \\
\end{tabular} & B1 & $\begin{array}{c}\text { (dn th)EODO-(s)IVS PF } \\
\text { AVG }\end{array}$ & $\mathrm{R} 2$ & (reparatio-reipvb) & & & 6 & 22 & 3.98 & & & \\
\hline 222 & $\begin{array}{c}\text { Ro-97-art } \\
143.379(29) \\
\end{array}$ & B1 & (...)S PF AVG & $\mathrm{R} 2$ & (reparatio-reipvb) & & & 12 & 22.7 & 5.20 & & & \\
\hline 223 & \begin{tabular}{|c|} 
Ro-97-art \\
$143.379(30)$ \\
\end{tabular} & B1 & & $\mathrm{R} 2$ & (reparatio-reipvb) & & & 12 & 21.5 & 4.54 & & & \\
\hline 224 & \begin{tabular}{|c|} 
Ro-97-art \\
$143.379(31)$
\end{tabular} & B1 & DN ARCADI-VS PF AVG & $\mathrm{R} 1$ & (glor)IA-(romanorvm) & & & 11 & 22 & 5.96 & & & \\
\hline 225 & $\begin{array}{c}\text { Ro-97-art } \\
143.379(32)\end{array}$ & B1 & D(n h)ONORIVS (pf avg) & $\mathrm{R} 1$ & (g)LORIA-(romanorvm) & & & 11 & 21.2 & 4.05 & & & \\
\hline 226 & \begin{tabular}{|c|} 
Ro-97-art \\
$143.379(34)$ \\
\end{tabular} & B1 & $\begin{array}{c}\text { (d)N ARCADI-VS PF } \\
\text { AV }(\mathrm{g})\end{array}$ & $\mathrm{R} 1$ & GLOR(ia)-ROMANO(rvm) & $\mathrm{S}(-)$ & & 11 & 22 & 4.06 & & & \\
\hline 227 & $\begin{array}{c}\text { Ro-97-art } \\
143.379(35) \\
\end{array}$ & B1 & DN A(r)CADI-VS (pf avg) & R1 & GLORIA-ROMANORVM & & & 6 & 23.5 & 4.30 & & & \\
\hline 228 & $\begin{array}{c}\text { Ro-97-art } \\
143.379(36)\end{array}$ & $\mathrm{B} 1$ & DN ARCADI-(vs pf avg) & & & & & & 20.9 & 5.72 & & & \\
\hline 229 & $\begin{array}{c}\text { Ro-97-art } \\
143.379(46)\end{array}$ & $\mathrm{B} 1$ & (dn) GRA(ti)A-(nvs pf avg) & & & & & & 23.5 & 3.66 & & & \\
\hline 230 & $\begin{array}{c}\text { Ro-97-art } \\
143.379(48)\end{array}$ & B1 & DN GRATIA-(nvs pf avg) & $\mathrm{R} 2$ & REPARATIO-(reipvb) & & $\mid \mathrm{S}$ & 6 & 23 & 4.99 & & & \\
\hline 231 & $\begin{array}{c}\text { Ro-97-art } \\
143.379(49) \\
\end{array}$ & B1 & DN GRATIA-NVS P(f avg $)$ & $\mathrm{R} 2$ & (re)PARAT(io)-REI(pvb) & $(-) \mathrm{N}$ & $\mid \mathrm{S}$ & 11 & 25.8 & 6.08 & & & \\
\hline 232 & \begin{tabular}{|c|} 
Ro-97-art \\
$143.379(52)$ \\
\end{tabular} & B1 & (dn gratia)-NVS PF AVG & $\mathrm{R} 2$ & (reparat)IO-REIP(vb) & & & 6 & 21 & 3.91 & & & \\
\hline 233 & $\begin{array}{c}\text { Ro-97-art } \\
143.379(58)\end{array}$ & B1 & $\begin{array}{l}\text { (dn) HONOR(iv)S P(f a ) } \\
\text { VG }\end{array}$ & $\mathrm{R} 1$ & (glo)RIA-(roman)ORVM & & & 6 & 21.5 & 4.46 & & & \\
\hline 234 & $\begin{array}{c}\text { Ro-97-art } \\
143.379(61)\end{array}$ & B1 & DN HONOR(ivs pf avg) & $\mathrm{R} 1$ & (glor)IA-ROMANO(rvm) & & & 5 & 19 & 4.46 & & & \\
\hline 235 & $\begin{array}{c}\text { Ro-97-art } \\
143.379(64) \\
\end{array}$ & B1 & DN HONOR(ivs pf avg) & $\mathrm{R} 1$ & (gloria)-(romano)RVM & & & 12 & 19.7 & 5.22 & & & \\
\hline 236 & \begin{tabular}{|c|} 
Ro-97-art \\
$143.379(69)$ \\
\end{tabular} & B1 & (dn) ARCA(di)-(vs pf avg) & $\mathrm{R} 1$ & (gloria)-ROMANOR(vm) & & & 11 & 20 & 4.36 & & & \\
\hline 237 & $\begin{array}{c}\text { Ro-97-art } \\
143.379(70)\end{array}$ & & (...) VS PF (avg) & $\mathrm{R} 1$ & (gloria)-ROM(anorv)M & & & & 21 & 3.71 & & & \\
\hline 238 & $\begin{array}{c}\text { Ro-97-art } \\
143.379(71)\end{array}$ & B4 & CONSTA-(ns pf avg) & R5 & (glo)RIA EX-(ercitvs) & & & 7 & 13.8 & 0.98 & $\begin{array}{l}i 337- \\
347 ?\end{array}$ & AE4 & \\
\hline 239 & \begin{tabular}{|c|} 
Ro-97-art \\
$143.379(72)$ \\
\end{tabular} & B1 & & & & & & & 15.2 & 1.26 & & & \\
\hline 240 & $\begin{array}{c}\text { Ro-97-art } \\
143.379(73) \\
\end{array}$ & & & & & & & & 21.8 & 3.05 & & & \\
\hline 241 & Sin registro & & Frustro & & Frustro & & & & 21 & 3.68 & & & \\
\hline 242 & Sin registro & & Frustro & & Frustro & & & & 19.5 & 4.25 & & & \\
\hline 243 & Sin registro & & Frustro & & Frustro & & & & 18.9 & 3.87 & & & \\
\hline 244 & Sin registro & & Frustro & & Frustro & & & & 20.9 & 5.70 & & & \\
\hline 245 & Sin registro & & Frustro & & Frustro & & & & 22.2 & 4.19 & & & \\
\hline
\end{tabular}

Figura 12: Conjunto monetario del siglo IV d.C. procedentes de la Calle Armiñán de Ronda. Catálogo de monedas por cecas. 


\section{REFERENCIAS}

Adroher Auroux, A., Aguayo de Hoyos, P. y Ruiz, C. (1993). Informe de la excavación de urgencia en el solar N. ${ }^{\circ} 5$ de la Calle Juan Bosco de Ronda. 1986. Anuario Arqueológico de Andalucía 1991, Tomo III, 407-411.

Aguayo de Hoyos, P., Castaño Aguilar, J. M. y Padial Robles, B. (2004). Análisis arqueológico y urbanístico de una manzana. Intervenciones de urgencia en el casco urbano de Ronda, 1994-2000. Anuario Arqueológico de Andalucía 2001, Tomo III/2, 772-787.

Castaño Aguilar, J. M., Aguayo de Hoyos, P., Delgado Blasco, P., Ordóñez Vergara, P., Padial Robles, B., y Weyh, K. (2005). Carta arqueológica municipal. Ronda: la ciudad. Sevilla: Dirección General de Bienes Culturales, Servicio de Investigación y Difusión del Patrimonio Histórico

Cepeda Ocampo, J. J. (2000). Maiorina Gloria Romanorum: monedas, tesoros y áreas de circulación en Hispania en el tránsito del siglo IV al V. Archivo Español de Arqueología, 73, 161-192.

Chaves Tristán, F., Chic García, G., Gil Fernández, R. (2000). El conjunto monetario de Cortijo Chirino, Écija (Sevilla). SPAL, 9, 465-475.

Mora Serrano, B. (1988). Reacuñaciones en la ceca de Acinipo. Acta Numismática, 17-18, 89-100.

Mora Serrano, B. (1990). Malaca, Acinipo y Lacipo: tres cecas monetales en los territorios malacitanos. Jábega, 67, 3-12.

Mora Serrano, B. (1993). La villa romana de Manguarra y San José, Cártama, Málaga. Estudio de los hallazgos monetarios. En Estudios dedicados a Alberto Balil in Memoriam (pp. 133-145). Málaga: Universidad de Málaga.

Mora Serrano, B. (1999). La circulación monetaria en la ciudad de Acinipo (Ronda, Málaga) y las comunicaciones entre el Estrecho y el Valle del Guadalquivir. En R. M. Sobral Centeno, M. ${ }^{a}$ P. García-Bellido y G. Mora (Eds.). Rutas, ciudades y moneda en Hispania (pp. 341-348). Anejos de Archivo Español de Arqueología, XX. Madrid: CSIC.

Mora Serrano, B. (2001). La circulación monetaria en los territorios malacitanos durante la Antigüedad. En F. Wulff Alonso, G. Cruz Andreotti y C. Martínez Maza. En Actas del II Congreso de Historia de Málaga: Comercio y comerciantes en la Historia Antigua de Málaga (siglos VIII a.C.-711 d.C.) (pp. 419-455). Málaga: Arguval.

Mora Serrano, B. (2003). Sobre la ocupación tardía del teatro romano de Malaca: los hallazgos monetarios antiguos. Mainake, 25, 359-376.

Mora Serrano, B. (2007). Moneda tardoantigua en Malaca (Málaga). Un ocultamiento monetario del siglo V d.C. procedente del teatro romano. Numisma, 251, 191-212.

Mora Serrano, B., y Ojeda Marín, M. (1988). Un tipo monetal de Acinipo y su relación con la numismática norteafricana. En E. Ripoll Perelló (Ed.). Actas del Congreso Internacional: El Estrecho de Gibraltar» (Ceuta, 1987), Tomo I (pp. 593-600). Madrid: Universidad Nacional de Educación a Distancia.

Morales Rodríguez, E. M. a (2000). Los municipios flavios de la Bética. (Tesis doctoral). Universidad de Granada. Granada. Recuperado de: http://hdl.handle.net/10481/4419

Nieto González, B. (2007). El mundo romano en la Depresión Natural de Ronda. En B. Nieto González, J. M. Castaño Aguilar y J. Padial Pérez (Eds.). Historia de Ronda. Desde la romanización a la época musulmana. I Congreso de Historia de Ronda (pp. 11-131). Ronda: Ayuntamiento de Ronda.

Ortiz Córdoba, J. (2015). Nuevos aportes para el conocimiento de la numismática de época tardía en Ronda (Málaga). Florentia Iliberritana, 26, 181-225.

Ortiz Córdoba, J. (2017). Aportes de la erudición local de los siglos XVI-XIX para el conocimiento de las ciudades romanas de Arvnda y Acinipo. En M. Ayarzagüena Sanz, G. Mora, y J. Salas Álvarez (Eds.). 150 años de Historia de la Arqueología. Teoría y Método de una disciplina (pp. 199-215). Madrid: Sociedad Española de Historia de la Arqueología.

Ortiz Córdoba, J. (En prensa). Novedades numismáticas en Acinipo: las monedas procedentes de las excavaciones de los años 80 en la mesa de Ronda la Vieja, Mainake, 37.

Rodríguez Oliva, P. (1979). El tesorillo de bronces bajoimperiales de Manilva (Málaga). En Actas del XV Congreso Nacional de Arqueología (pp. 835-852). Zaragoza: Secretaría General de los Congresos Arqueológicos Nacionales, Universidad de Zaragoza.

San Vicente, J. I. (1999). Circulación monetaria en Hispania durante el siglo IV d.C. Madrid: Museo Casa de la Moneda. 